TRANSACTIONS OF THE

AMERICAN MATHEMATICAL SOCIETY

Volume 356, Number 6, Pages 2405-2441

S 0002-9947(03)03385-3

Article electronically published on November 12, 2003

\title{
STABLE REPRESENTATIVES FOR SYMMETRIC AUTOMORPHISMS OF GROUPS AND THE GENERAL FORM OF THE SCOTT CONJECTURE
}

\author{
MIHALIS SYKIOTIS
}

\begin{abstract}
Let $G$ be a group acting on a tree $X$ such that all edge stabilizers are finite. We extend Bestvina-Handel's theory of train tracks for automorphisms of free groups to automorphisms of $G$ which permute vertex stabilizers. Using this extension we show that there is an upper bound depending only on $G$ for the complexity of the graph of groups decomposition of the fixed subgroups of such automorphisms of $G$.
\end{abstract}

\section{INTRODUCTION}

Let $G$ be a group acting on a tree $X$. We study automorphisms $f$ of $G$ for which there is a map (not necessarily isometry) $\tilde{f}: X \longrightarrow X$, called representative of $f$, such that $\tilde{f}(g x)=f(g) \tilde{f}(x)$ for every $g \in G$ and $x \in V(X)$. Here we will call such automorphisms symmetric. In the most interesting cases, it is easy to see that an automorphism $f$ of $G$ is symmetric if and only if it permutes vertex stabilizers, provided that no vertex stabilizer is contained in an edge stabilizer. This equivalent characterization, together with the Bass-Serre theory, for which the reader is referred to [9] and [14, allows us to find examples of groups in which each automorphism is symmetric. In particular the class of groups with this property, i.e. each automorphism is symmetric, contains all free products of freely indecomposable groups, all free by finite groups, and all accessible groups including finitely presented groups with infinite ends.

In [15] it was shown that if $G$ is a group acting on a tree $X$ with finite quotient graph, finite edge stabilizers and $f$ is a symmetric endomorphism of $G$, then, being a subgroup of $G$, the fixed subgroup $F i x(f)=\{g \in G: f(g)=g\}$ of $f$ admits a finite graph of groups decomposition. In [2] Bestvina and Handel proved the Scott conjecture which says that the rank of the fixed subgroup Fix $(f)$ of a free group automorphism $f$ is at most $n$, by using an algorithm which for any topological representative of $f$ produces a stable relative train track one. In view of these results, it is natural to ask if there is an upper bound for the "complexity" of the graph of groups decomposition of the fixed subgroups of symmetric automorphisms of $G$.

On the other hand, as the existence of stable relative train track representatives for automorphisms of free groups has also proven to be useful in the study of the

Received by the editors July 24, 2002 and, in revised form, April 17, 2003.

2000 Mathematics Subject Classification. Primary 20E36, 20E08, 20 E06.

(C)2003 American Mathematical Society 
outer automorphism group $\operatorname{Out}\left(F_{n}\right)$ of a free group $F_{n}$ of finite rank $n$ (see [3], [4] and [5]), it would be nice to develop the corresponding theory for symmetric automorphisms of groups. It should be noted here that the Bestvina-Handel algorithm is presented in 10 by Dicks and Ventura in a special form (they work with the top stratum only) in the language of groupoids for injective endomorphisms of free groups of finite rank.

The purpose of this paper is to present an extension of the Bestvina-Handel theory for automorphisms of free groups to symmetric automorphisms of groups acting on trees such that all edge stabilizers are finite with the same cardinality on the one hand, and to generalize the Scott conjecture to groups acting on trees and symmetric automorphisms of them on the other.

In order to generalize the Scott conjecture for symmetric automorphisms of groups acting on trees, first we need to introduce the notion of the complexity of a graph of groups, since each action without inversions of a group $G$ on a tree $X$ defines a graph of groups with fundamental group isomorphic to $G$ and conversely; each graph of groups gives an action without inversions of his fundamental group on a tree, as insures the main result of the Bass-Serre theory.

Let $(\mathcal{G}, Y)$ be a finite graph of groups whose underlying graph $Y$ has fundamental group (topologically) a free group of $\operatorname{rank} r(Y)$. A vertex $v$ of $Y$ is called degenerate if the vertex group $G_{v}$ is equal to an edge group $G_{e}$ for some edge $e$ whose endpoint is the vertex $v$. The complexity of $(\mathcal{G}, Y)$ (and consequently of $G$ with respect to this splitting) is defined to be the sum $r(Y)+V_{d}(Y)$, where $V_{d}(Y)$ denotes the number of nondegenerate vertices of $Y$. It is our belief that the complexity is the appropriate measure for the fundamental group of a graph of groups, since the complexity of a free group $F$ of rank $r(F)$ is its rank $r(F)$, while the complexity of a free product $*_{i=1}^{n} G_{i}$ with nontrivial factors is $n$. Note also, that the complexity of a graph of groups is invariant under elementary operations as these described in Section 3.

We can now state the first of our main results related to the complexity of a symmetric automorphism fixed subgroup.

Theorem 6.12. Let $G$ be the fundamental group of a finite graph of groups $(\mathcal{G}, Y)$ such that all edge stabilizers are finite with the same cardinality, let $|H|$. If $f$ is a symmetric automorphism of $G$ (that is, $f$ maps nondegenerate vertex groups onto conjugates of themselves), then the subgroup Fix $(f)$ inherits from $G$ a splitting of complexity at most $C(Y)|H|$.

Theorem 6.6 has some very interesting consequences.

Proposition 6.15. Let $G=*_{H} G_{i}$ be the free product of $G_{i}$ 's, $i=1, \ldots, n$, with amalgamated subgroup $H$ properly contained in each factor $G_{i}$. Supppose that $H$ is a finite group and that each factor $G_{i}$ has at most one end. If $f \in \operatorname{Aut}(G)$, then the subgroup Fix $(f)$ inherits from $G$ a splitting of complexity at most $n|H|$.

Corollary $6.17([8])$. Let $G=*_{i=1}^{n} G_{i}$ be the free product of $G_{i}$ 's, $i=1, \ldots, n$, where each factor $G_{i}$ is indecomposable with respect to free products and let $f$ be an automorphism of $G$. Then the subgroup Fix $(f)$ has complexity at most $n$.

Using the accessibility of finitely presented groups and an inductive argument, we obtain from Theorem 6.12 the most general result concerning the complexity of Fix $(f)$ of an automorphism $f$ of a finitely presented group $G$. 
Theorem 6.18. Let $G$ be a finitely presented group with infinite ends and let $(\mathcal{G}, Y)$ be a graph of groups decomposition of $G$ of minimal number of vertices such that all vertex groups have at most one end and all edge groups are finite. If $f \in A u t(G)$, then there is a positive integer $n=n(G)$ such that Fix $(f)$ admits a graph of groups decomposition with vertex and edge groups of the form Fix $(f) \cap g G_{x} g^{-1}, g \in G$, $x \in Y$, of complexity at most $n$.

The paper is organized as follows. In Section 2, for the reader's convenience, we first review some definitions and basic facts from the Perron-Frobenius theory for irreducible matrices. Then, we represent every symmetric endomorphism $f$ of a group $G$ acting on a tree $X$ by an endomorphism $\tilde{f}$ of the fundamental groupoid $\pi X$ of $X$ and define the transition matrix $M(\tilde{f})$ of $\tilde{f}$. Thus, to each representative we can assign a finite sequence of irreducible matrices (the irreducible diagonal blocks of $M(\tilde{f}))$ whose Perron-Frobenius eigenvalues measure the efficiency of $\tilde{f}$.

In Section 3, we describe the basic operations on $G$-trees which are needed in order to improve the efficiency of such a representative $\tilde{f}$. In Section 4 , we present the extension of the main notions and results of Bestvina-Handel's train track theory to representatives of symmetric automorphisms. In particular we prove the existence of stable relative train track representatives for symmetric automorphisms of groups acting on trees with finite edge stabilizers which further have the same cardinality. The main theorem for stable relative train track representatives, in our point of view, is presented in Section 5 .

Theorem 5.4. Suppose that $\tilde{f}: X \longrightarrow X$ is a stable relative train track representative of $f$ and that $H_{r}$ is an exponentially growing stratum. Then, there is at most one $G$-orbit of indivisible Nielsen paths in $X_{r}$ that contain edges of $H_{r}$.

Finally, in Section 6 we consider certain properties of the complexity and give the proofs of our results that provide bounds on the complexity of fixed subgroups.

\section{Preliminaries}

2.1. Irreducible matrices and Perron-Frobenius theory. In this subsection we record what we need in our analysis from the Perron-Frobenius theory faithfully following [10].

Definitions 2.1. A permutation matrix is an $n \times n$ matrix with 1 in the $(\sigma(i), i)$ th position for each $i=1, \ldots, n$ and all other entries equal to 0 , where $\sigma$ is a permutation of $\{1, \ldots, n\}$. A reducible matrix is a square matrix $n \times n, M$ which by simultaneous permutation of rows and columns can take the form

$$
\left[\begin{array}{ll}
A & B \\
O & C
\end{array}\right]
$$

where the matrices $A, B, O, C$ have size $k \times k, k \times(n-k),(n-k) \times k$, $(n-k) \times(n-k)$, respectively, $1 \leq k<n$. Thus, the matrix $M$ is reducible if and only if there is a permutation matrix $P$ such that

$$
P M P^{-1}=\left[\begin{array}{cc}
A & B \\
O & C
\end{array}\right]
$$

A square matrix is called irreducible if it is not reducible. For two matrices $M$ and $N$ with the same size, we write $M \leq N$ if $\mu_{i j} \leq \nu_{i j}$ for all $i, j$. 
We now review some basic facts about irreducible matrices. For their proofs we refer the reader to [10].

Lemma 2.2. Let $M$ be a nonzero, nonnegative real square matrix. The matrix $M$ is irreducible if and only if $\sum_{k=1}^{m} M^{k}>0$ for some $m \geq 1$.

Proposition 2.3. Suppose that $0 \neq M=\left(\mu_{i j}\right)$ is an irreducible $n \times n$ matrix of nonnegative integers, $w$ is a nonzero, nonnegative, real column vector and $\mu$ is a nonnegative real number. Then:

(i) There is a unique positive, real, right eigenvector $v$ of norm $1\left(\|v\|_{1}=1\right)$ for $M$ which has associated eigenvalue $\lambda(M) \geq 1$.

(ii) If $\lambda(M)=1$, then $M$ is a permutation matrix.

(iii) $\mu_{i j} \leq \lambda(M)^{n}$ for any $i, j$.

(iv) If $M w \leq \mu w$, then $\lambda(M) \leq \mu$.

The eigenvector $v$ is called a normalized Perron-Frobenius right eigenvector of $M$ and the associate eigenvalue, denoted by $P F(M)$, a Perron-Frobenius eigenvalue.

Let $N^{\prime}$ and $N$ be square integer matrices. We say that $N^{\prime}$ is dominated by $N$ if there is a submatrix $N^{\prime \prime}$ of $N$ such that $N^{\prime} \leq N^{\prime \prime}$.

Proposition 2.4. (i) Let $N$ and $N^{\prime}$ be irreducible nonnegative integer square matrices such that $N^{\prime}$ is dominated by $N$. Then $P F\left(N^{\prime}\right) \leq P F(N)$. If further $P F\left(N^{\prime}\right)=P F(N)$, then $N^{\prime}=N$.

(ii) Suppose that $K$ and $L$ are nonnegative integer matrices with no zero rows and columns, respectively. If $L K$ is irreducible, then $K L$ is irreducible as well and $P F(K L)=P F(L K)$.

2.2. Symmetric endomorphisms of groups acting on trees and their matrices. In this paper we are interested in actions of groups $G$ on trees with finite quotient graph. For a graph $Y$, we write $V(Y)$ and $E(Y)$ respectively for the vertex set and edge set.

Definition 2.5. The endomorphism $f$ of $G$ is called symmetric if there exists a $G$-tree $X$ and a map $\tilde{f}: V(X) \longrightarrow V(X)$ such that $\tilde{f}(g v)=f(g) \tilde{f}(v)$ for each $g \in G$ and $v \in V(X)$.

Remark 2.6. If $v_{1}, \ldots, v_{n}$ is a set of representatives for the action of $G$ on the set of vertices, then $f$ is symmetric if and only if there are $g_{i} \in G$ for $i=1, \ldots, n$ and $j(i) \in\{1, \ldots, n\}$ such that $f\left(G_{v_{i}}\right) \subseteq g_{i} G_{v_{j(i)}} g_{i}^{-1}$. In fact, it is clear that this condition is necessary. In order to see that it is sufficient we define the map $\tilde{f}: X \longrightarrow X$ by $\tilde{f}\left(g v_{i}\right)=f(g) g_{i} v_{j(i)}$. The map $\tilde{f}$ is well defined and thus $f$ is symmetric.

Let $\pi X$ be the fundamental groupoid (homotopy-classes of paths) on $X$. We know that every homotopy class contains a unique reduced path. We denote by [q] the unique reduced path homotopic to $q$. The homotopy relation is denoted by $\simeq$.

The pair $(\tilde{f}, X)$ is called representative of $f$, although usually the tree $X$ will be omitted. The map $\tilde{f}$ can be extended to an endomorphism of the fundamental groupoid $\pi X$ of $X$ as follows: $\tilde{f}(p)=[\tilde{f}(i p), \tilde{f}(t p)]$, where $[u, v]$ denotes the unique geodesic from the vertex $u$ to the vertex $v$, and $i p, t p$ are the initial and terminal vertices of the path $p$, respectively. For $p, q \in \pi X$, we write $p \circ q$ for the composition of the paths $p$ and $q$ if no cancellation occurs; otherwise we write $p \cdot q$. Note that $G$ 
acts on $\pi X$ and $\tilde{f}(g p)=f(g) \tilde{f}(p)$. If $p=e_{1} \circ \cdots \circ e_{n}$, then $\tilde{f}(p) \simeq \tilde{f}\left(e_{1}\right) \cdots \tilde{f}\left(e_{n}\right)$; this holds since $\tilde{f}\left(e_{1}\right) \cdots \tilde{f}\left(e_{n}\right)$ is a path in the tree $X$ which has the same initial and terminal vertices with $\tilde{f}(p)$.

In [15] it has been proved that if $f$ is a symmetric endomorphism of $G$, then the subgroup Fix $(f)$ is the fundamental group of a finite graph of groups such that all edge groups are finite while the vertex groups are either of the form $F i x\left(\left.f\right|_{G_{v}}\right)$, where $v$ is a vertex of $X$ fixed by $\tilde{f}$ or finite. Also in [15] it is shown that the above generalizes all previous results in this direction as [7], [11, 12] and [13].

Let $E(X)=\bigsqcup_{i=1}^{i=n} O_{i}$, where $O_{i}=G e_{i}$ for $e_{i} \in E(X)$. That is, $O_{i}$ is the orbit of the edge $e_{i}, i=1, \ldots, n$.

Definition 2.7. The transition matrix $M(\tilde{f})$ associated to $\tilde{f}: X \longrightarrow X$ is the $n \times n$ matrix whose $(i, j)$-th entry is the number of times the path $\tilde{f}\left(e_{i}\right)$ crosses the orbit $O_{j}$, regardless of orientation.

Remarks 2.8. The matrix $M(\tilde{f})$ is an $n \times n$ matrix of nonnegative integers which is independent of the representatives $e_{i}$ because the action of $G$ on $X$ is by isometries. If the map $\tilde{f}$ is an isometry, then $M(\tilde{f})$ is a permutation matrix. It is not difficult to see that if $f$ and $g$ are symmetric with representatives $\tilde{f}, \tilde{g}: X \longrightarrow X$ such that $f=g$ in $\operatorname{Out}(G)=\operatorname{Aut}(G) / \operatorname{Inn}(G)$, then $M(\tilde{f})=M(\tilde{g})$.

Definitions 2.9. Let $X_{0}$ be a subgraph of a $G$-tree $X$ and $f$ a symmetric endomorphism of $G$. The subgraph $X_{0}$ is called an $\tilde{f}$-invariant $G$-subgraph of $X$ if the following conditions are satisfied:

(1) $X_{0}$ is $G$-subset of $X$, i.e. $E\left(X_{0}\right)=\bigsqcup_{i \in I_{0}} O_{i}$, where $I_{0} \subseteq I=\{1, \ldots, n\}$.

(2) $\tilde{f}\left(X_{0}\right) \subseteq \pi X_{0}$.

For $p \in \pi X$ we write $[p]_{X_{0}}$ for the row vector obtained from $p$ by deleting the coordinates $i \in I_{0}$. Similarly we write $(M(\tilde{f}))_{X_{0}}$ for the submatrix of $M(\tilde{f})$ corresponding to the orbits of $I \backslash I_{0}$. A filtration for a symmetric endomorphism $f$ is an increasing sequence $\emptyset=X_{0} \subseteq X_{1} \subseteq \ldots \subseteq X_{m}=X$ of $\tilde{f}$-invariant $G$-subgraphs of the tree $X$. The $G$-set of the edges $X_{i} \backslash X_{i-1}$ is called $i$-stratum and is denoted by $H_{i}$. A maximal filtration is one for which each of the matrices $M_{i}=M\left(\tilde{f} \mid X_{i}\right)_{X_{i-1}}$ is irreducible. By simultaneous permutations of rows and columns the matrix $M(\tilde{f})$ can be put in the form

$$
\left[\begin{array}{cccc}
M_{k} & * & \cdots & * \\
0 & M_{k-1} & \cdots & * \\
\vdots & \vdots & \ddots & \vdots \\
0 & \cdots & 0 & M_{1}
\end{array}\right]
$$

where the matrices $M_{i}$ are zero $1 \times 1$ or irreducible matrices and are determined up to row-column permutation; therefore the Perron-Frobenius eigenvalues of $M_{i}$ 's depend only on the matrix $M(\tilde{f})$. It is clear that the above decomposition of $M(\tilde{f})$ defines a maximal filtration $\emptyset=X_{0} \subseteq X_{1} \subseteq \ldots \subseteq X_{k}=X$ for which $M\left(\tilde{f} \mid X_{i}\right)_{X_{i-1}}=M_{i}$. We write $H_{i}<H_{j}$ if $X_{i} \subseteq X_{j}$. As in [2] we call the stratum $H_{i}$ exponentially growing if $\lambda_{i}>1$, where $\lambda_{i}$ is the Perron-Frobenius eigenvalue of $M_{i}$. We also define $\Lambda=\Lambda(M(\tilde{f}))$ to be the nonincreasing sequence $\lambda_{i_{1}} \geq \lambda_{i_{2}} \geq \ldots \geq \lambda_{i_{k}}$ of Perron-Frobenius eigenvalues of exponentially growing strata. Finally, we equip the set of sequences $\Lambda=\{\Lambda(M(\tilde{f})), \tilde{f}$ representative of $f\}$ with the lexicographic order (where units are added at the end if this is necessary). 


\section{The ELEMENTARY OPERATIONS}

Let $G$ be a group which acts on a tree $X$ and $f$ an automorphism of $G$. In this section, given a representative $(\tilde{f}, X)$ of $f$ we describe the appropriate operations and its effect on $(\tilde{f}, X)$ that are used in the algorithm which starts with $(\tilde{f}, X)$ and finds an efficient representative (see the proof of Theorem 4.22). To insure that the algorithm terminates and that the operations do not change the complexity we must make some assumptions on $(\tilde{f}, X)$.

We recall that the vertex $v$ of $X$ is called degenerate if $G_{v}=G_{e}$ for some edge $e$ of $X$ for which $v \in\{i(e), t(e)\}$. We see that if the vertex $v$ is degenerate, so is each vertex in the orbit $G v$ of $v$.

Hypotheses 3.1. Unless otherwise stated, in this paper we consider representatives $(\tilde{f}, X)$ of $f$ such that the following conditions are verified:

(i) The edge groups are finite and have the same cardinality.

(ii) If $u$ and $v$ are nondegenerate vertices, then $\tilde{f}(u) \neq \tilde{f}(v)$.

(iii) $f$ permutes nondegenerate vertex groups.

Thus, from now on, we call an automorphism $f$ of $G$ symmetric if there is a representative of $f$ such that the three conditions above are verified.

Remark 3.2. Although condition (iii) implies condition (ii) it is more convenient to put it separately. To see this, suppose that $\tilde{f}(u)=\tilde{f}(v)$ for the nondegenerate vertices $u$ and $v$. The fact that $\tilde{f}$ is a representative of $f$ yields $f\left(G_{u}\right) \subseteq G_{\tilde{f}(u)}$ and $f\left(G_{v}\right) \subseteq G_{\tilde{f}(v)}$. From this we deduce that the vertices $\tilde{f}(u)$ and $\tilde{f}(v)$ are nondegenerate. By condition (iii), there are nondegenerate vertices $x, y$ such that $f\left(G_{u}\right)=G_{x}$ and $f\left(G_{v}\right)=G_{y}$. Now, since the stabilizers of the nondegenerate vertices $x$ and $y$ are contained in $G_{\tilde{f}(u)}$, it follows that $x=y=\tilde{f}(u)$ and thus $f\left(G_{u}\right)=f\left(G_{v}\right)$. Hence, from the injectivity of $f$ we take $G_{u}=G_{v}$ which shows that $u=v$.

Remark 3.3. We must keep in mind that all the following hold in our analysis whenever condition (i) is verified, all nondegenerate vertex groups are finite with the same cardinality and $f$ is an injective symmetric endomorphism of $G$; then automatically conditions (ii) and (iii) are verified. But, in the proofs below we consider only the first case because of the careful handling of the arguments that are required.

We start our analysis with the following lemma.

Lemma 3.4. Let $\tilde{f}: X \longrightarrow X$ be a representative of the automorphism $f$ as above.

(1) For any edge $e$ of $X$ with $\tilde{f}(e)$ a vertex, the image of $e$ in the quotient graph $X / G$ is not a loop.

(2) Suppose that the edges $e_{1}$ and $e_{2}$ with $i\left(e_{1}\right)=i\left(e_{2}\right)$ belong to different orbits and that $\tilde{f}\left(e_{1}\right)=\tilde{f}\left(e_{2}\right)$. Then the vertices $t\left(e_{1}\right)$ and $t\left(e_{2}\right)$ belong to different orbits as well.

Proof. (1) Assume that there exists $g$ in $G$ such that $g i(e)=t(e)$; in particular $g$ has infinite order being a generator of $\pi_{1}(X / G)$. Since $\tilde{f}(i(e))=\tilde{f}(t(e))$ the element $f(g)$ stabilizes the vertex $\tilde{f}(i(e))$; therefore the vertex $\tilde{f}(i(e))$ is nondegenerate and hence from condition (iii) $g$ also stabilizes a vertex, say $v$. Now we observe that 
$d(v, i(e)) \neq d(v, t(e))=d(v, g i(e))$, which gives a contradiction since the group $G$ acts on $X$ by isometries.

(2) As before if $g$ is an element of $G$ such that $g t\left(e_{1}\right)=t\left(e_{2}\right)$, then the element $g$ is of infinite order and since $\tilde{f}\left(t\left(e_{1}\right)\right)=\tilde{f}\left(t\left(e_{2}\right)\right)$, it stabilizes a nondegenerate vertex, say again $v$. We denote by $u$ the vertex of the set $\left\{i\left(e_{1}\right), t\left(e_{1}\right), t\left(e_{2}\right)\right\}$ of minimum distance from $v$ and by $p$ the reduced path $[v, u]$ from $v$ to $u$. In the case where $u=i\left(e_{1}\right)$, the element $g$ stabilizes the vertex $u$ [14, Corollary 2, p. 62]. It follows that $g e_{1}=e_{2}$ which contradicts our hypothesis. On the other hand, if $u=t\left(e_{1}\right)$ we have $p \circ e_{1}^{-1} \circ e_{2}=\left[v, t\left(e_{2}\right)\right]=g p$ which is improper. Similarly, we obtain a contradiction if $u=t\left(e_{1}\right)$.

In the sequel we modify a definition from [10].

Definition 3.5. We call an edge $e$ of $X$ a $\tilde{f}$-fixed point which is not a vertex if the path $\tilde{f}(e)$ contains $e^{\epsilon}, \epsilon \in\{-1,1\}$ and in the case that $\epsilon=1, e$ is neither the first nor the last edge of $\tilde{f}(e)$. We will say that the $\tilde{f}$-fixed point $e$ which is not a vertex is contained in the orbit $O_{i}$ if $e \in O_{i}$. Since $X$ is a tree, every $\tilde{f}$-fixed point $e$ which is not a vertex uniquely determines a natural number - the "coordinate" of the path $\tilde{f}(e)$ in which the edge $e^{\epsilon}$ appears. Consequently the set of $\tilde{f}$-fixed points $e$ which are not vertices in the orbit $O_{i}$ determines a subset of $\{1, \ldots,|\tilde{f}(e)|\}$ denoted by $A_{i}$. We define $\operatorname{FPnV}(\tilde{f})=\sum_{i=1}^{n}\left|A_{i}\right|$.

The next remark will be proved useful in the proofs of this section.

Remark 3.6. Two $\tilde{f}$-fixed points $e$ and $g e$ in the same orbit $O_{i}$ which are not vertices determine the same coordinate if and only if $g^{-1} f(g) \in G_{e}$.

3.1. The collapse of a $\tilde{f}$-trivial edge. This is a special case of the collapse of a connected graph of groups $(\mathcal{G}, Y)$ along a subgraph $Z$ of $Y$ (for details see [1]).

Definitions 3.7. An edge $e$ of a tree $X$ is called $\tilde{f}$-trivial if $\tilde{f}(e)$ is a vertex; that is, $\tilde{f}(i(e))=\tilde{f}(t(e))$. It is clear that if the edge $e$ is $\tilde{f}$-trivial, so is each edge in the orbit of $e$. In this case condition (ii) implies that at least one of the vertices $i(e)$ and $t(e)$ must be degenerate, say $t(e)$. Since we assume that $t(e)$ is a degenerate vertex, we conclude that each connected component $X_{i}, i \in I$, of the orbit $O_{e}$ has either the form $\left\{g(x e), g \in G_{i(x e)}\right\}$ or is a path (may be infinite) depending on whether $i(e)$ is nondegenerate or not. Let us denote by $\pi: X \longrightarrow X^{\prime}$ the projection which collapses each $X_{i}$ to a point. It is easy to check that $X^{\prime}$ is a $G$-tree and that $\pi$ is a $G$-map. From Lemma 3.4 we know that the edge $e$ is not a loop in the quotient graph $X / G$. This implies that $r(X / G)=r\left(X^{\prime} / G\right)$. Also the stabilizer of the vertex $v=\pi(x)$ is $G_{x}$ if $x$ is not contained in $O_{e}$ and $G_{x i(e)}$ if $x$ is contained in the component of $x i(e)$. Thus condition (iii) holds for $X^{\prime}$ while the edge groups remain the same.

We define a new representative $\tilde{f}^{\prime}: X^{\prime} \longrightarrow X^{\prime}$ as follows. Let $p$ be a reduced path in $X^{\prime}$. Then $p$ has the form $\pi\left(e_{1}\right) \circ \pi\left(e_{2}\right) \circ \cdots \circ \pi\left(e_{n}\right)$ where the edges $e_{i}$ do not belong to the orbit $O_{e}$. Since $t\left(\pi\left(e_{i}\right)\right)=i\left(\pi\left(e_{i+1}\right)\right)$ the vertices $t\left(e_{i}\right)$ and $i\left(e_{i+1}\right)$ for $i=1, \ldots, n-1$ must lie in the same component $X_{j(i)}, j \in I$; therefore there is a unique path $q_{i}$ in $X_{j(i)}$ which connects them. We set $\tilde{p}=e_{1} \circ q_{1} \circ \cdots \circ q_{n-1} \circ e_{n}$ and define $\tilde{f}^{\prime}(p)$ to be the unique reduced path homotopic to $\pi \circ \tilde{f}(\tilde{p})$. Since $e$ is $\tilde{f}$ trivial the map $\tilde{f}^{\prime}: \pi X^{\prime} \longrightarrow \pi X^{\prime}$ does not depend on the lifting $\tilde{p}$, and it is easy to check that $\tilde{f}^{\prime}$ is in fact a representative of $f$. We have already seen that $X^{\prime}$ satisfies 
conditions (i) and (iii). Condition (ii) is satisfied by $\tilde{f}^{\prime}$ because the projection $\pi: X \longrightarrow X^{\prime}$ does not identify nondegenerate vertices. If the representative $\tilde{f}$ determines the maximal filtration $\emptyset=X_{0} \subseteq X_{1} \subseteq \ldots \subseteq X_{m}=X$, then the sequence $\emptyset=\pi\left(X_{0}\right) \subseteq \pi\left(X_{1}\right) \subseteq \ldots \subseteq \pi\left(X_{m}\right)=X^{\prime}$ is a filtration for $\left(\tilde{f}^{\prime}, X^{\prime}\right)$, the matrix $M\left(\tilde{f}^{\prime}\right)$ is obtained from $M(\tilde{f})$ by deleting the rows and columns corresponding to the edges of $O_{e}$, and the strata of the maximal filtration for $\tilde{f}^{\prime}: X^{\prime} \longrightarrow X^{\prime}$ arise from those of $\tilde{f}: X \longrightarrow X$.

Proposition 3.8. Suppose that $\tilde{f}^{\prime}: X^{\prime} \longrightarrow X^{\prime}$ is obtained from $\tilde{f}: X \longrightarrow X$ by collapsing the $\tilde{f}$-trivial edge e. Then $\Lambda^{\prime} \leq \Lambda$ and $F \operatorname{PnV}\left(\tilde{f}^{\prime}\right) \leq F \operatorname{PnV}(\tilde{f})$.

Proof. Any matrix $M_{i}^{\prime}$ of the maximal filtration for $\tilde{f}^{\prime}$ is obtained from some $M_{j(i)}$ by deleting some rows and columns. Thus, by Proposition 2.4 we have $\Lambda^{\prime} \leq \Lambda$. It is clear that if the edge $\pi(x)$ is a $\tilde{f}^{\prime}$-fixed point not a vertex, so is the edge $x$, while $G_{\pi(x)}=G_{x}$. Hence $\pi(x)$ and $\pi(g x)$ determine the same coordinate if and only if $x$ and $g x$ do (Remark 3.6). It follows that $F \operatorname{PnV}\left(\tilde{f}^{\prime}\right) \leq F \operatorname{PnV}(\tilde{f})$.

\subsection{Valence one homotopy.}

Definitions 3.9. Let $v$ be a degenerate vertex of $X$ of valence one (this is equivalent to saying that the vertex $[v]$ has valence one in $X / G$ ), and let $e$ be the edge incident to $v$. Then each vertex $g v$ has valence one as well, and it is clear that the subgraph $X^{\prime}=X \backslash O_{e}$ is a $G$-invariant subtree of $X$. Moreover each connected component $X_{i}$ of $X \backslash X^{\prime}$ has only one vertex in common with the tree $X^{\prime}$, let $v_{i}$. Let $\pi: X \longrightarrow X^{\prime}$ be the projection which collapses each component $X_{i}$ to the vertex $v_{i}$. Then, $\pi$ is a $G$-map and we define $\tilde{f}^{\prime}: X^{\prime} \longrightarrow X^{\prime}$ by $\tilde{f}^{\prime}(p)=[\pi \circ \tilde{f}(p)]$. As in the collapse of a $\tilde{f}$-trivial edge, we see that $\tilde{f}^{\prime}$ is a representative of $f$ satisfying conditions (i), (ii) and (iii). In this case we say that $\tilde{f}^{\prime}: X^{\prime} \longrightarrow X^{\prime}$ is obtained from $\tilde{f}: X \longrightarrow X$ by a valence one homotopy.

The proof of the following proposition is exactly as that of Proposition 3.8.

Proposition 3.10. Suppose that $\tilde{f}^{\prime}: X^{\prime} \longrightarrow X^{\prime}$ is obtained from $\tilde{f}: X \longrightarrow X$ by a valence one homotopy. Then $\Lambda^{\prime} \leq \Lambda$ and $F \operatorname{Pn} V\left(\tilde{f}^{\prime}\right) \leq F \operatorname{PnV}(\tilde{f})$.

3.3. Subdividisions. We first consider the general form of subdivision.

Definitions 3.11. Let $e$ be an edge of $X$ such that $\tilde{f}(e)=p_{1} \circ p_{2}$; then $\tilde{f}(g e)=$ $f(g) p_{1} \circ f(g) p_{2}$. Let $X^{\prime}$ be the tree obtained from $X$ by subdividing the orbit $O_{e}$ into two orbits. More specifically, we add a new vertex $v_{g}$ to each edge $g e$ in the orbit $O_{e}$ such that $g e=e_{1 g} \circ e_{2 g}, t\left(e_{1 g}\right)=i\left(e_{2 g}\right)=v_{g}$ and set $e_{i}=e_{i 1_{G}}$. The action of $G$ on $X^{\prime}$ is defined by $x e_{i g}=e_{i x g}$ and thus $G_{e_{i}}=G_{e}$.

We can think of $\pi X$ as a subgroupoid of $\pi X^{\prime}$ via the injection $j: \pi X \longrightarrow \pi X^{\prime}$, where the map $j$ is given by $j(g e)=e_{1 g} \circ e_{2 g}$ in the orbit $O_{e}$ and is the identity elsewhere. We define $\tilde{f}^{\prime}: \pi X^{\prime} \longrightarrow \pi X^{\prime}$ by the rule $\tilde{f}^{\prime}\left(e_{i g}\right)=f(g) p_{i}$ for $i=1,2$ and $\tilde{f}^{\prime}=\tilde{f}$ on the other orbits. The map $\tilde{f}^{\prime}$ determines a representative of the automorphism $f$ and we say that $\tilde{f}^{\prime}: X^{\prime} \longrightarrow X^{\prime}$ is obtained from $\tilde{f}: X \longrightarrow X$ by subdivision using $\tilde{f}(e)=p_{1} \circ p_{2}$.

Proposition 3.12. Suppose that $\tilde{f}^{\prime}: X^{\prime} \longrightarrow X^{\prime}$ is obtained from $\tilde{f}: X \longrightarrow X$ by subdivision as above. Then $\Lambda^{\prime}=\Lambda$ and $F \operatorname{Pn} V\left(\tilde{f}^{\prime}\right) \leq F \operatorname{PnV}(\tilde{f})$. 
Proof. Let $\emptyset=X_{0} \subseteq X_{1} \subseteq \ldots \subseteq X_{m}=X$ be the maximal filtration associated to the representative $\tilde{f}$. Suppose that the edge $e$ belongs to the stratum $H_{i}$. Then the subdivision has no effect on the strata $H_{j}$ as well as on their matrices $M_{j}$ for $j \neq i$. We only need to check what happens in the stratum $H_{i}$. The matrix of the injection $j$ is $M_{i}(j)=\left[\begin{array}{lll}I & 0 & 0 \\ 0 & 1 & 1\end{array}\right]$, where the last row of $M(j)$ corresponds to $O_{e}$, and the last two columns to the orbits $O_{e_{1}}$ and $O_{e_{2}}$. Assuming that the paths $p_{1}$ and $p_{2}$ cross the orbit $O_{e}, m_{1}$ and $m_{2}$ times respectively, we have $M_{i}(\tilde{f})=\left[\begin{array}{cc}A & A \\ C_{1}+C_{2} & m_{1}+m_{2}\end{array}\right]$ and $M_{i}\left(\tilde{f}^{\prime}\right)=\left[\begin{array}{ccc}A & L & L \\ C_{1} & m_{1} & m_{1} \\ C_{2} & m_{2} & m_{2}\end{array}\right]$, where the last row and column of $M(\tilde{f})$ correspond to $O_{e}$, and the two last rows and columns of $M\left(\tilde{f}^{\prime}\right)$ correspond to $O_{e_{1}}$ and $O_{e_{2}}$, respectively. Note that $L$ is a column vector while $C_{1}$ and $C_{2}$ are row vectors. If $\tilde{f}^{\prime}\left(e_{1}\right)$ (similar for $\tilde{f}^{\prime}\left(e_{2}\right)$ ) is entirely into $X_{i-1}$, then $C_{1}=\overrightarrow{0}, m_{1}=0$, the orbit $O_{e_{1}}$ determines a new stratum with zero martix and the filtration $\emptyset=X_{0}^{\prime} \subseteq \ldots \subseteq$ $X_{i-1}^{\prime} \subseteq\left(X_{i-1}^{\prime} \cup O_{e_{1}}\right) \subseteq\left(X_{i}^{\prime} \backslash O_{e_{1}}\right) \subseteq \ldots \subseteq X_{m}^{\prime}=X^{\prime}$ is a maximal filrtation for $\tilde{f}^{\prime}$, where $X_{k}^{\prime}=j\left(X_{k}\right)$. In this case, the matrix of the stratum $\left(X_{i}^{\prime} \backslash O_{e_{1}}\right)$ is exactly $M_{i}$ and hence $\Lambda^{\prime}=\Lambda$. So we can suppose that

$$
\begin{aligned}
& \tilde{f}^{\prime}\left(e_{1}\right) \text { and } \tilde{f}^{\prime}\left(e_{2}\right) \text { is not entirely into } X_{i-1} \text {; that } \\
& \text { is } C_{1} \neq \overrightarrow{0} \text { or } m_{1}>0 \text {, and } C_{2} \neq \overrightarrow{0} \text { or } m_{2}>0 .
\end{aligned}
$$

We also note that one of the matrices $C_{1}$ and $C_{2}$ is not the zero matrix because of the irreducibility of $M_{i}(\tilde{f})$.

We will show that the matrix $M_{i}\left(\tilde{f}^{\prime}\right)$ is irreducible and hence the filtration $\emptyset=$ $X_{0}^{\prime} \subseteq X_{1}^{\prime} \subseteq \ldots \subseteq X_{m}^{\prime}=X^{\prime}$ is maximal. First, we observe that

$$
M_{i}(\tilde{f}) M_{i}(j)=M_{i}(j) M_{i}\left(\tilde{f}^{\prime}\right) .
$$

Therefore $M_{i}^{k}(\tilde{f}) M_{i}(j)=M_{i}(j) M_{i}^{k}\left(\tilde{f}^{\prime}\right)$ for every positive integer $k$ and consequently $\left(\sum_{k=1}^{m} M_{i}^{k}(\tilde{f})\right) M_{i}(j)=M_{i}(j)\left(\sum_{k=1}^{m} M_{i}^{k}\left(\tilde{f}^{\prime}\right)\right)$. If $m$ is a positive integer such that $\sum_{k=1}^{m} M_{i}^{k}(\tilde{f})>0$, then it is easy to see that $\left(\sum_{k=1}^{m} M_{i}^{k}(\tilde{f})\right) M_{i}(j)>0$. If $P_{1}, \ldots, P_{n}$ are the rows of $\left(\sum_{k=1}^{m} M_{i}^{k}\left(\tilde{f}^{\prime}\right)\right)$, then the rows of $M_{i}(j)\left(\sum_{k=1}^{m} M_{i}^{k}\left(\tilde{f}^{\prime}\right)\right)$ are $P_{1}, \ldots, P_{n-1}+P_{n}$ with $P_{i}>0$ for $i=1, \ldots, n-2$ and $P_{n-1}+P_{n}>0$. Suppose that the first zero in the rows $P_{n-1}, P_{n}$ appears in the $r$-th column. Then from (I) we conclude that the matrix $M_{i}\left(\tilde{f}^{\prime}\right)\left(\sum_{k=1}^{m} M_{i}^{k}\left(\tilde{f}^{\prime}\right)\right)$ has no zeros before the $r+1$-th column. This shows how we can find a positive sum of powers of $M_{i}\left(\tilde{f}^{\prime}\right)$ which means that $M_{i}\left(\tilde{f}^{\prime}\right)$ is irreducible.

If $\lambda^{\prime}=P F\left(M_{i}\left(\tilde{f}^{\prime}\right)\right), \lambda=P F\left(M_{i}(\tilde{f})\right)$ and $l^{\prime}, l$ are the corresponding normalized Perron-Frobenius eigenvectors, then $M_{i}(\tilde{f}) M_{i}(j) l^{\prime}=M_{i}(j) M_{i}\left(\tilde{f}^{\prime}\right) l^{\prime}=M_{i}(j)\left(\lambda^{\prime} l^{\prime}\right)$ and hence $M_{i}(j) l^{\prime}$ is a Perron-Frobenius eigenvector of $M_{i}(\tilde{f})$ which has norm 1. It follows that $M_{i}(j) l^{\prime}=l$ and $M_{i}(\tilde{f}) l=\lambda^{\prime} l=\lambda l$. Thus $\lambda^{\prime}=\lambda$ and $\Lambda^{\prime}=\Lambda$.

To prove that $F \operatorname{Pn} V\left(\tilde{f}^{\prime}\right) \leq F \operatorname{Pn} V(\tilde{f})$, we first note that any $p \in \pi X^{\prime}$ is mapped under $\tilde{f}^{\prime}$ into the groupoid $\pi X$ and the $\tilde{f}^{\prime}$-fixed points not vertices which do not belong to the orbits $O_{e_{1}}$ and $O_{e_{2}}$ are exactly the $\tilde{f}$-fixed points not vertices which do not belong to the orbit $O_{e}$. Let $A$ be the set of the coordinates of the $\tilde{f}$-fixed points not vertices in the orbit $O_{e}$. We write $A=A_{1} \sqcup A_{2}$, where $A_{1} \subseteq\left\{1, \ldots,\left|p_{1}\right|\right\}$ and $A_{2} \subseteq\left\{\left|p_{1}\right|+1, \ldots,\left|p_{1}\right|+\left|p_{2}\right|\right\}$. We leave it to the reader to verify that each $\tilde{f}^{\prime}$-fixed point not a vertex $g e_{i}$ for $i=1,2$ gives us a $\tilde{f}$-fixed point not a vertex $g e$ on $A_{i}$. Since $G_{e}=G_{e_{i}}$ we have $g^{-1} f(g) \in G_{e}$ iff $g^{-1} f(g) \in G_{e_{i}}$ and this proves that $\operatorname{FPnV}\left(\tilde{f}^{\prime}\right) \leq F \operatorname{Pn} V(\tilde{f})$. 
Remarks 3.13. Suppose the edge $e=e_{1} \circ e_{2}$ belongs to the exponentially growing stratum $H_{i}$. If both of $\tilde{f}\left(e_{1}\right)$ and $\tilde{f}\left(e_{2}\right)$ are not contained in $X_{i-1}$, then as we have already seen in the above proof $H_{i}^{\prime}=\left(H_{i} \backslash O_{e}\right) \cup\left\{O_{e_{1}}, O_{e_{2}}\right\}$ is an exponentially growing stratum such that $\left|H_{i}^{\prime}\right|=\left|H_{i}\right|+1$. In the other case, without loss of generality we can suppose that $\tilde{f}\left(e_{1}\right) \notin X_{i-1}$ while $\tilde{f}\left(e_{2}\right) \in X_{i-1}$; then $H_{i}^{\prime}=$ $\left(H_{i} \backslash O_{e}\right) \cup O_{e_{1}}$ is again an exponentially growing stratum, and $\left|H_{i}^{\prime}\right|=\left|H_{i}\right|$. In any case the stratum $H_{i}$ produces only one exponentially growing stratum $H_{i}^{\prime}$ whose transition matrix is denoted by $M_{i}^{\prime}$.

Let $H_{i}$ be an exponentially growing stratum and $l$ a Perron-Frobenius eigenvector of $M_{i}$. If $l^{\prime}$ is the Perron-Frobenius eigenvector of $M^{\prime}$ for which $\left\|l^{\prime}\right\|_{1}=\|l\|_{1}$, then as in the proof of Proposition 3.12 we see that $M_{i}(j) l^{\prime}=l$; therefore $l(x)=l^{\prime}(x)$ for $x \neq e$ and $l(e)=l^{\prime}\left(e_{1}\right)+l^{\prime}\left(e_{2}\right)$, where $l(x)$ is the coordinate of $l$ corresponding to the orbit $O_{x}$.

Now we are dealing with a specific form of subdivision which is used to reduce the number $F \operatorname{Pn} V(\tilde{f})$.

Definitions 3.14. Let $e \in E(X)$ be a $\tilde{f}$-fixed point not a vertex; that is, $\tilde{f}(e)=$ $p_{1} \circ e^{\epsilon} \circ p_{2}$ with $\epsilon \in\{-1,+1\}$, and if $\epsilon=1$, then $p_{1}$ and $p_{2}$ are nontrivial paths. As before $X^{\prime}$ is the tree obtained from $X$ by subdividing each ge into two edges $e_{1 g}$ and $e_{2 g}$, the corresponding new vertex is denoted by $v_{g}$, and the stabilizers of $e_{1 g}, e_{2 g}$ and $v_{g}$ are $G_{g e}$. Again we view $\pi X$ as a subgroupoid of $\pi X^{\prime}$ via the monomorphism $j: \pi X \longrightarrow \pi X^{\prime}$, where $j(g e)=e_{1 g} \circ e_{2 g}$ (as usual we shall omit the $\operatorname{map} j)$.

Let $x_{1}$ and $x_{2}$ be two edges of $X^{\prime}$ for which $e^{\epsilon}=x_{1} \circ x_{2}$. We define the map $\tilde{f}^{\prime}: \pi X^{\prime} \longrightarrow \pi X^{\prime}$ as follows: $\tilde{f}^{\prime}\left(e_{1 g}\right)=f(g) p_{1} \circ f(g) x_{1}, \tilde{f}^{\prime}\left(e_{2 g}\right)=f(g) x_{2} \circ f(g) p_{2}$, and $\tilde{f}^{\prime}$ is $\tilde{f}$ on $X \backslash O_{e}$. It is immediate that $\tilde{f}^{\prime}(g p)=f(g) \tilde{f}^{\prime}(p)$ for $p \in \pi X^{\prime}$. We also note that $\tilde{f}^{\prime}(e)=\tilde{f}(e)$. In this case, we say that the representative $\tilde{f}^{\prime}: X^{\prime} \longrightarrow X^{\prime}$ is obtained from $\tilde{f}: X \longrightarrow X$ by subdividing at the $\tilde{f}$-fixed point $e$ which is not a vertex.

Proposition 3.15. Suppose that $\tilde{f}^{\prime}: X^{\prime} \longrightarrow X^{\prime}$ is obtained from $\tilde{f}: X \longrightarrow$ $X$ by subdividing at the $\tilde{f}$-fixed point $e$ which is not vertex. Then $\Lambda^{\prime}=\Lambda$ and $F \operatorname{Pn} V\left(\tilde{f}^{\prime}\right)<F \operatorname{Pn} V(\tilde{f})$.

Proof. As in the proof of Proposition 3.12 the subdvision has no effect on the transition matrices of the strata not containing the orbit $O_{e}$. Suppose that $e \in$ $H_{i}$. Then $M_{i}(j)=\left[\begin{array}{ccc}I & 0 & 0 \\ 0 & 1 & 1\end{array}\right]$ and if $p_{1}$ and $p_{2}$ cross the orbit $O_{e} m_{1}$ and $m_{2}$-times respectively, then $M_{i}(\tilde{f})=\left[\begin{array}{cc}A & L \\ c_{1}+c_{2} & m_{1}+m_{2}+1\end{array}\right]$. In the case in which $\epsilon=1$ the transition matrix of $j\left(H_{i}\right)$ has the form

$$
M_{i}\left(\tilde{f}^{\prime}\right)=\left[\begin{array}{ccc}
A & L & L \\
c_{1} & m_{1}+1 & m_{1} \\
c_{2} & m_{2} & m_{2}+1
\end{array}\right]
$$

and

$$
M_{i}\left(\tilde{f}^{\prime}\right)=\left[\begin{array}{ccc}
A & L & L \\
c_{1} & m_{1} & m_{1}+1 \\
c_{2} & m_{2}+1 & m_{2}
\end{array}\right]
$$

otherwise, where $I$ denotes the identity matrix, the last row and last column of $M(\tilde{f})$ correspond to $O_{e}$, and the two last rows and columns of $M_{i}\left(\tilde{f}^{\prime}\right)$ correspond 
to $O_{e_{1}}$ and $O_{e_{2}}$, respectively. In any case we have $M_{i}(\tilde{f}) \cdot M_{i}(j)=M_{i}(j) \cdot M_{i}\left(\tilde{f}^{\prime}\right)$ and the same arguments as in the proof of Proposition 3.12 imply that $\Lambda^{\prime}=\Lambda$.

The subdivision does not create new fixed points on the orbits different from $O_{e}$, so we must see what happens to the orbit $O_{e}$. If ge is a $\tilde{f}$-fixed point not a vertex which determines the same "coordinate" with $e$, then $\tilde{f}(g e)=f(g) p_{1} \circ g e^{\epsilon} \circ f(g) p_{2}$ and $g^{-1} f(g) \in G_{e}$. Clearly all these fixed points disappear after subdivision. Let $g e_{1}$ be a $\tilde{f}^{\prime}$-fixed point not a vertex in the orbit $O_{e_{1}}$. If $\epsilon=1$ (the same arguments work as well in the case where $\epsilon=-1)$, then $\tilde{f}^{\prime}\left(g e_{1}\right)=f(g) p_{1} \circ f(g) e_{1}$ and $\left(g e_{1}\right)^{ \pm 1}$ cannot occur in the end of the path $\tilde{f}^{\prime}\left(g e_{1}\right)$. Therefore $g e_{1}$ occurs in $f(g) p_{1}$. Since $X^{\prime}$ is obtained from $X$ by subdividing the orbit $O_{e}$, it follows that $g e_{2}$ occurs in $f(g) p_{1}$ beside (in fact after) $g e_{1}$. This implies that the edge $g e$ occurs in $f(g) p_{1}$ and is a fixed point not a vertex in $O_{e}$. Similarly, for each $\tilde{f}^{\prime}$-fixed point not a vertex $g e_{2}$ in the orbit $O_{e_{2}}$ we find a $\tilde{f}$-fixed point $g e$ in the orbit $O_{e}$ which occurs in $\underset{\tilde{f}}{f}(g) p_{2}$. Thus each $\tilde{f}^{\prime}$-fixed point not a vertex in the orbits $O_{e_{1}}$ and $O_{e_{2}}$ gives us a $\tilde{f}$-fixed point not a vertex in the orbit $O_{e}$, while $G_{e}=G_{e_{1}}=G_{e_{2}}$.

We close this subsection by describing another form of subdivision, the so-called core subdivision.

Definitions 3.16. Suppose that there are at least two strata for $\tilde{f}: X \longrightarrow X$ and that $H_{i}$ is an exponentially growing stratum. Then, since for each $e \in H_{i}$ we have $\tilde{f}(e) \in \pi X_{i}$ while $\tilde{f}(e) \notin \pi X_{i-1}$, there exists a path $p_{e 2}$ whose first and last edges belong to $H_{i}$ and paths $p_{e 1}$ and $p_{e 3}$ in $\pi X_{i-1}$ such that $\tilde{f}(e)=p_{e 1} \circ p_{e 2} \circ p_{e 3}$. Let $X^{\prime}$ be the tree obtained by subdividing the orbit $O_{e}$ into three orbits for all $e \in H_{i}$, i.e. $g e=e_{1 g} \circ e_{2 g} \circ e_{3 g}$, for all $e \in H_{i}$. We set $e_{i 1_{G}}=e_{i}$ for $i=1,2,3$ and define the action of $G$ on $X^{\prime}$ by $h e_{i g}=e_{i h g}$. Note that the stabilizer of any edge $e_{i g}$ is $G_{g e}$ as are also the stabilizers of the vertices $v_{1 g}=i\left(e_{2 g}\right)$ and $v_{2 g}=i\left(e_{3 g}\right)$. We view $\pi X$ as a subgroupoid of $\pi X^{\prime}$ via the map $j: \pi X \longrightarrow \pi X^{\prime}$, where $j(g e)=e_{1 g} \circ e_{2 g} \circ e_{3 g}$. Let $X_{i-1}^{\prime 1}=j\left(X_{i-1}\right) \cup\left\{O_{e_{1}} \cup O_{e_{3}}, e \in H_{i}\right\}$. Then, we can write each path $p_{e 2}$ as a composition $p_{e 2}=p_{e 21} \circ p_{e 22} \circ p_{e 23}$, where the paths $p_{e 21}$ and $p_{e 23}$ lie in $\pi X_{i-1}^{\prime 1}$ and the first and last edges of $p_{e 22}$ lie in $j\left(X_{i}\right) \backslash X_{i-1}^{\prime 1}$. Now, we define the representative $\tilde{f}^{\prime}$ by $\tilde{f}^{\prime}\left(e_{1 g}\right)=f(g)\left(p_{e 1} \circ p_{e 21}\right), \tilde{f}^{\prime}\left(e_{2 g}\right)=f(g) p_{e 22}, \tilde{f}^{\prime}\left(e_{3 g}\right)=f(g)\left(p_{e 23} \circ p_{e 3}\right)$, and $\tilde{f}^{\prime}(y)=\tilde{f}(y)$ for $y \notin H_{i}$. In this case, we say that $\tilde{f}^{\prime}: X^{\prime} \longrightarrow X^{\prime}$ is obtained from $\tilde{f}: X \longrightarrow X$ by the core subdivision on the stratum $H_{i}$.

Proposition 3.17. Suppose that $\tilde{f}^{\prime}: X^{\prime} \longrightarrow X^{\prime}$ is obtained from $\tilde{f}: X \longrightarrow X$ by the core subdivision on the stratum $H_{i}$. Then $\Lambda^{\prime}=\Lambda$ and $F \operatorname{Pr} V\left(\tilde{f}^{\prime}\right) \leq F \operatorname{Pn} V(\tilde{f})$.

Proof. We consider the filtration $\emptyset \subseteq X_{0}^{\prime} \subseteq \ldots \subseteq X_{i-1}^{\prime} \subseteq X_{i-1}^{\prime 1} \subseteq X_{i}^{\prime} \subseteq \ldots \subseteq X^{\prime}$, where $X_{k}^{\prime}=j\left(X_{k}\right)$. Let $H_{i}^{\prime}=X_{i}^{\prime} \backslash X_{i-1}^{\prime 1}$ and let $H_{i-1}^{\prime 1}=X_{i-1}^{\prime 1} \backslash X_{i-1}^{\prime}$. The subdivision has no effect on the other strata and thus we must check the effect on these two strata. We first observe that the path $p_{e 21}$ (similarly for $p_{e 23}$ ) is one of the edges $y_{1}$ and $y_{3}^{-1}$, where $y \in H_{i}$ is the first edge of $p_{e 2}$. This means that the stratum $H_{i-1}^{\prime 1}$ has matrix (not necessarily irreducible) consisting of units and zeros and hence does not add exponentially growing strata in the maximal filtration which is obtained from the above one. For the same reason the orbit $O_{e_{2}}$ occurs as many times in $\tilde{f}^{\prime}\left(y_{2}\right)$ as the orbit $O_{e}$ in $\tilde{f}(y)$ does. This shows that the transition matrix of $\tilde{f}^{\prime}$ on $H_{i}^{\prime}$ is exactly the transition matrix of $\tilde{f}$ on $H_{i}$ and thus $\Lambda^{\prime}=\Lambda$.

Regarding fixed points it suffices to look at the orbits of $H_{i}$ and $j\left(H_{i}\right)$. If $e \in H_{i}$ the path $\tilde{f}^{\prime}\left(g e_{1}\right)$ - except from the last edge-lies in $\pi X_{i-1}$; hence if $g e_{1}$ is a $\tilde{f}^{\prime}$-fixed 
point not a vertex, we must have $f(g) p_{e 21}=g e_{1}^{-1}$. This leads to a contradiction, since the first edge of $p_{e 2}$ is one of $y_{1}$ and $y_{3}^{-1}, y \in H_{i}$. Thus the orbits $O_{e_{1}}$ for $e \in H_{i}$ do not contain $\tilde{f}^{\prime}$-fixed points not vertices. Similarly, we see that the same is true for the orbits $O_{e_{3}}$, for $e \in H_{i}$. Now, if $g e_{2}$ is a $\tilde{f}^{\prime}$-fixed point not a vertex, then it is easy to see that $g e$ is also a $\tilde{f}$-fixed point not a vertex. Moreover, $g e_{2}$ and $h e_{2}$ determine the same coordinate iff $g e$ and he do.

Remark 3.18. The proof of the preceding proposition shows that $H_{i}^{\prime}$ is a stratum of the maximal filtration for $\tilde{f}^{\prime}$ with $\left|H_{i}^{\prime}\right|=\left|H_{i}\right|$, and that for each $y \in H_{i}^{\prime}$ the first and last edges of $\tilde{f}^{\prime}(y)$ are contained in $H_{i}^{\prime}$.

Remark 3.19. Suppose $\tilde{f}^{\prime}: X^{\prime} \longrightarrow X^{\prime}$ is obtained from $\tilde{f}: X \longrightarrow X$ by any form of subdivision. Subdivisions create only degenerate vertices and do not change the quotient graph. In particular condition (i) is satisfied after subdivisions. Moreover, since the representative $\tilde{f}^{\prime}$ is $\tilde{f}$ on the vertices of $X$, it follows that conditions (ii) and (iii) are satisfied for $\tilde{f}^{\prime}$ as well.

3.4. Folding. We first recall the definition and properties of the Stallings folding construction general in $G$-graphs.

Definition 3.20. Suppose that $X$ is a $G$ graph and that $x, y$ are two edges of $X$ with the same initial vertex; that is, $i(x)=i(y)$. When the edges $x$ and $y^{-1}$ belong to distinct orbits we say that we have the fold $(x, y)$. If $(x, y)$ is a fold we can identify $x$ with $y$ and $\tau(x)$ with $\tau(y)$ in the $G$-sets $E(X)$ and $V(X)$, respectively. The result is again a $G$-graph denoted by $X /[x=y]$.

The following well-known result (for a proof we refer to [6, Lemma 8.36]) is important because it insures that the category of $G$-trees is closed under the folding operations.

Lemma 3.21. Let $(x, y)$ be a fold in a $G$-tree $X$. Then $X /[x=y]$ is again a G-tree.

Remarks 3.22. The natural map $\pi: X \longrightarrow X /[x=y]$ is a $G$-map. Let $(x, y)$ be a fold. In the case that $x$ and $y$ belong to distinct orbits, the stabilizer of the edge $\pi(x)=\pi(y)$ is the subgroup generated by $G_{x}$ and $G_{y}$ in $G_{i(x)}$. On the other hand, if $x=g y$, then $G_{\pi(x)}=\left\langle g, G_{x}\right\rangle \subseteq G_{i(x)}$. Similarly one can describe the stabilizer $G_{\pi(t(x))}=G_{\pi(t(y))}$ of $\pi(t(x))$.

Definitions 3.23. Let $\tilde{f}: X \longrightarrow X$ be a representative of $f$ and let $e_{1}$ and $e_{2}$ be a pair of not $\tilde{f}$-trivial (otherwise we collapse these) distinct edges of the tree $X$ in the strata $H_{i}$ and $H_{j}$, respectively, such that $i\left(e_{1}\right)=i\left(e_{2}\right)$ and $\tilde{f}\left(e_{1}\right)=\tilde{f}\left(e_{2}\right)$. Then $\tilde{f}\left(g e_{1}\right)=f(g) \tilde{f}\left(e_{1}\right)=f(g) \tilde{f}\left(e_{2}\right)=\tilde{f}\left(g e_{2}\right)$ for each $g \in G$. Under the above hypothesis we say that the map $\tilde{f}$ admits the fold $\left(e_{1}, e_{2}\right)$. Without loss of generality, we can suppose that $e_{1}$ and $e_{2}^{-1}$ belong to distinct orbits; otherwise we subdivide the edges $e_{1}$ and $e_{2}$.

We define $X^{\prime}=X /\left[e_{1}=e_{2}\right]$. From Lemma $3.21 X^{\prime}$ is a $G$-tree. Let $\varphi$ : $\pi X^{\prime} \longrightarrow \pi X$ be the map which factors $\tilde{f}$, i.e. $\varphi(\pi(e))=\tilde{f}(e)$. The map $\varphi$ is well defined, since the relation $\pi(e)=\pi\left(e^{\prime}\right)$ implies that $\tilde{f}(e)=\tilde{f}\left(e^{\prime}\right)$, and is an $f$-equivariant $G$-map (this means that $\varphi(g x)=f(g) \varphi(x)$ for $x \in X)$. We also define $\tilde{f}^{\prime}: \pi X^{\prime} \longrightarrow \pi X^{\prime}$ by $\tilde{f}^{\prime}\left(p^{\prime}\right)=\left[\pi \circ \varphi\left(p^{\prime}\right)\right]$. Then $\tilde{f}^{\prime} \circ \pi(p) \simeq \pi \circ \tilde{f}(p)$ and $\tilde{f}^{\prime}\left(g p^{\prime}\right)=f(g) \tilde{f}^{\prime}\left(p^{\prime}\right)$ for $p \in \pi X$ and $p^{\prime} \in \pi X^{\prime}$; in particular $\tilde{f}^{\prime}$ is a representative of 
$f$. In this case we say that $\tilde{f}^{\prime}: X^{\prime} \longrightarrow X^{\prime}$ is obtained from $\tilde{f}: X \longrightarrow X$ by folding the edges $e_{1}$ and $e_{2}$. If moreover $H_{i}=H_{j}$, we say that the folding takes place in the stratum $H_{i}$.

We continue with some simple but essential observations. From condition (ii), it follows that one of the vertices $t\left(e_{1}\right)$ and $t\left(e_{2}\right)$ must be degenerate, say $t\left(e_{1}\right)$. Also, from condition (i), we have $f\left(G_{e_{1}}\right)=G_{\tilde{f}\left(e_{1}\right)}=f\left(G_{e_{2}}\right)$ and hence $G_{e_{1}}=G_{e_{2}}$ (even if $f$ is a monomorphism). If we suppose that $e_{1}$ and $e_{2}$ lie in the same orbit, then $e_{1}=g e_{2}$ for some $g \in G$ and so $f(g)$ stabilizes the path $\tilde{f}\left(e_{1}\right)$; therefore $g \in G_{e_{2}}$, which is improper (the same argument shows that if the maximal common segment of $\tilde{f}\left(e_{1}\right)$ and $\tilde{f}\left(e_{2}\right)$ is not a vertex, then $e_{1}$ and $e_{2}$ belong to different orbits). From these and Lemma 3.4(2), we conclude that the edge and vertex groups in $X^{\prime}$ are exactly as in $X$, and since the folding map $\pi$ does not identify nondegenerate vertices, conditions (i), (ii) and (iii) are satisfied by $\left(\tilde{f}^{\prime}, X^{\prime}\right)$, while Lemma 3.4 insures that the graph $X^{\prime} / G$ has the same rank with $X / G$.

Proposition 3.24. Suppose $\tilde{f}^{\prime}: X^{\prime} \longrightarrow X^{\prime}$ is obtained from $\tilde{f}: X \longrightarrow X$ by folding the edges $e_{1}$ and $e_{2}$. If the folding takes place in an exponentially growing stratum $H_{i}$, then $\Lambda^{\prime} \leq \Lambda$. In all other cases $\Lambda^{\prime}=\Lambda$, while always $F \operatorname{PnV}\left(\tilde{f}^{\prime}\right) \leq F \operatorname{PnV}(\tilde{f})$.

Before we prove the proposition we shall make some conventions. First, we can suppose that every edge of $X$ is not $\tilde{f}$-trivial; otherwise we collapse all $\tilde{f}$-trivial edges of $X$ in view of Proposition 3.8 (every $\tilde{f}$-trivial edge constitutes a stratum with zero matrix). If $\left|\tilde{f}\left(e_{1}\right)\right|=\left|\tilde{f}\left(e_{2}\right)\right|=1$ it is clear that $O_{e_{1}} \neq O_{e_{2}^{-1}}$, since $G$ acts on $X$ without inversions. In the case where $\left|\tilde{f}\left(e_{1}\right)\right|=\left|\tilde{f}\left(e_{2}\right)\right| \geq 2$ and $O_{e_{1}}=O_{e_{2}^{-1}}$ we subdivide the orbits $O_{e_{1}}$ and $O_{e_{2}}$ such that the new tree has no $\tilde{f}^{\prime}$-trivial edges and apply Proposition 3.12. Thus, in the proof below, we can suppose that the $\operatorname{martix} M(\tilde{f})$ has no zero rows and that $O_{e_{1}} \neq O_{e_{2}^{-1}}$.

Proof. We begin by proving that $F \operatorname{Pn} V\left(\tilde{f}^{\prime}\right) \leq F \operatorname{Pn} V(\tilde{f})$. Let $y$ be a $\tilde{f}^{\prime}$-fixed point not a vertex of $X^{\prime}$ with $\tilde{f}^{\prime}(y)=q_{1} \circ y^{\epsilon} \circ q_{2}$. Note that the map $\pi$ is locally surjective, thus every path in $\pi X^{\prime}$ has a lifting in $\pi X$; also $\pi$ is injective on the edges which do not belong to the orbits $O_{e_{1}}$ and $O_{e_{2}}$. Set $e=\pi\left(e_{1}\right)=\pi\left(e_{2}\right)$. If $y \notin O_{e}$, then there exists a unique edge $x$ such that $\pi(x)=y, G_{x}=G_{y}$ and easily $x$ is a $\tilde{f}$-fixed point not a vertex. Suppose now that $y=g e$. Then from the definition of $\tilde{f}^{\prime}$ we conclude that $\varphi(g e)=p_{1} \circ g e_{i}^{\epsilon} \circ p_{2}$ for some $i \in\{1,2\}$, where $p_{1}$ and $p_{2}$ are liftings of $q_{1}$ and $q_{2}$, respectively. Thus $\tilde{f}\left(g e_{i}^{\epsilon}\right)=\varphi \circ \pi\left(g e_{i}^{\epsilon}\right)=\varphi\left(g e^{\epsilon}\right)=p_{1} \circ g e_{i}^{\epsilon} \circ p_{2}$ and hence $g e_{i}^{\epsilon}$ is a $\tilde{f}$-fixed point not a vertex on $X$. Also, since $G_{e}=G_{e_{i}}$, if $g e$ and $x e$ are fixed points not vertices which do not determine the same coordinate, then $g e_{i}^{\epsilon}$ and $x e_{i}^{\epsilon}$ are, too.

Let $\emptyset=X_{0} \subseteq \ldots \subseteq X_{i} \subseteq \ldots \subseteq X_{m}=X$ be the maximal filtration determined by $\tilde{f}$. If $X_{i}^{\prime}=\pi\left(X_{i}\right)$, then $\emptyset=X_{0}^{\prime} \subseteq \ldots \subseteq X_{i}^{\prime} \subseteq \ldots \subseteq X_{m}^{\prime}=X$ is a filtration for $\tilde{f}^{\prime}$, from which we will obtain a maximal one. Suppose that $H_{i}$ and $H_{j}$ are the strata of $e_{1}$ and $e_{2}$, respectively. Since $\tilde{f}^{\prime} \circ \pi \simeq \pi \circ \tilde{f}$ and $X$ is a tree, the map $\pi$ in any path $\tilde{f}(p)$ removes only expresions of the form $g e_{1} \circ g e_{2}^{-1}$ or $g e_{1}^{-1} \circ g e_{2}$. In particular if $x$ is an edge of the stratum $H_{l}$ for $l \neq i, j$, then $\tilde{f}^{\prime}$ does not change the number of the appearances of the orbits $O_{y}$ in $\tilde{f}^{\prime}(\pi(x))$ for $y \notin H_{i}, H_{j}$. Therefore the folding has no effect on the matrices of the strata $H_{l}$ for $l \neq i, j$. 
If $i \neq j$, and $j>i$ (similarly if $i>j$ ), then the matrix $M_{j}$ has a zero row. From this and the irreducibility of $M_{j}$ we conclude that $H_{j}=O_{e_{2}}$ and $M_{j}=0$. Particularly the stratum $H_{j}$ is not exponentially growing. Moreover the matrix $M_{i}^{\prime}$ is exactly $M_{i}$, since $\tilde{f}\left(e_{1}\right)$ does not contain edges of the orbit $O_{e_{2}}$. This proves that $\Lambda^{\prime}=\Lambda$ when the folding takes place in different strata.

We now consider the case in which the folding takes place in $H_{i}$; that is, $i=j$. We note that the matrices of the other strata are irreducible since they do not change. Suppose the path $\tilde{f}\left(e_{1}\right)$ crosses the orbits $O_{e_{1}}$ and $O_{e_{2}} m_{1}$ and $m_{2}$-times, respectively. Then

$$
M_{i}(\varphi)=\left[\begin{array}{ccc}
A & L_{1} & L_{2} \\
C & m_{1} & m_{2}
\end{array}\right], \quad M_{i}(\tilde{f})=\left[\begin{array}{ccc}
A & L_{1} & L_{2} \\
C & m_{1} & m_{2} \\
C & m_{1} & m_{2}
\end{array}\right]
$$

and

$$
M_{i}(\pi)=\left[\begin{array}{ll}
I & 0 \\
0 & 1 \\
0 & 1
\end{array}\right]
$$

where the last rows and columns of $M_{i}(\tilde{f})$ correspond to orbits $O_{e_{1}}$ and $O_{e_{2}}$, respectively (similarly for $M_{i}(\pi)$ and $M(\varphi)$ ). If we set $N=M_{i}(\varphi) M_{i}(\pi)=\left[\begin{array}{cc}A & L_{1}+L_{2} \\ C & m_{1}+m_{2}\end{array}\right]$, then we observe $\left(\pi\right.$ being injective on $E(X) \backslash\left(O_{e_{1}} \cup O_{e_{2}}\right)$ ) that the matrices $M_{i}\left(\tilde{f}^{\prime}\right)$ and $M_{i}(\varphi) M_{i}(\pi)$ differ on the last column only which corresponds to $O_{e}$. In particular, inequality holds because expresions of the form $g\left(e_{1} \circ e_{2}^{-1}\right)^{ \pm 1}$ can appear in the path $\tilde{f}(y)$ for $y \in E(X)$. Thus $M_{i}(\varphi) M_{i}(\pi) \geq M_{i}(\pi \varphi)=M_{i}\left(\tilde{f}^{\prime}\right)$, where inequality occurs in the last column.

Since $M_{i}(\tilde{f})=M_{i}(\pi) M_{i}(\varphi)$ is irreducible, Proposition 2.4 implies that $N$ is irreducible as well and $P F\left(M_{i}(\tilde{f})\right)=P F(N)$. Now, each irreducible submatrix $N^{\prime}$ of $M_{i}\left(\tilde{f}^{\prime}\right)$ is dominated by $N$. Hence, by Proposition 2.4 we have $P F\left(N^{\prime}\right) \leq P F(N)$. This shows that if the stratum $H_{i}$ is not exponentially growing, then the folding in $H_{i}$ does not produce any exponentially growing strata and therefore $\Lambda=\Lambda^{\prime}$. Moreover, if $\operatorname{PF}\left(N^{\prime}\right)=\operatorname{PF}\left(M_{i}(\tilde{f})\right)$, then $N^{\prime}=N$; in particular $M_{i}\left(\tilde{f}^{\prime}\right)$, being equal to $N$, is an irreducible matrix and the filtration $\emptyset=X_{0}^{\prime} \subseteq \ldots \subseteq X_{i}^{\prime} \subseteq \ldots \subseteq X_{m}^{\prime}=X$ is maximal. It follows that $\Lambda=\Lambda^{\prime}$ in this case. The only case in which $\Lambda^{\prime}<\Lambda$ is when $P F\left(N^{\prime}\right)<P F\left(M_{i}(\tilde{f})\right)$.

Remark 3.25. Suppose that the folding takes place in an exponentially growing stratum $H_{i}$ and that $\Lambda=\Lambda^{\prime}$. Then as we have shown before $M_{i}\left(\tilde{f}^{\prime}\right)=N$, the stratum $H_{i}^{\prime}$ is exponentially growing and $\left|H_{i}\right|=\left|H_{i}^{\prime}\right|+1$. Let $l$ be a Perron-Frobenius right eigenvector of $M_{i}(\tilde{f})$ and let $l^{\prime}$ be the Perron-Frobenius right eigenvector of $M_{i}\left(\tilde{f}^{\prime}\right)$ for which $\left\|l^{\prime}\right\|_{1}+l^{\prime}(e)=\|l\|_{1}{ }_{1}^{1}$ where $l^{\prime}(e)$ denotes the entry of $l^{\prime}$ corresponding to the orbit of $e$. Then, since $M_{i}(\tilde{f}) M_{i}(\pi) l^{\prime}=M_{i}(\pi) M_{i}(\varphi)\left(M_{i}(\pi) l^{\prime}=M_{i}(\pi) M_{i}\left(\tilde{f}^{\prime}\right) l^{\prime}=\right.$ $\operatorname{PF}\left(M_{i}\left(\tilde{f}^{\prime}\right)\right) M_{i}(\pi) l^{\prime}$ and $\left\|M_{i}(\pi) l^{\prime}\right\|_{1}=\|l\|_{1}$ we deduce that $M_{i}(\pi) l^{\prime}=l$ and hence $l\left(e_{1}\right)=l^{\prime}(e)=l\left(e_{2}\right)$.

Remark 3.26. Let $e_{1}$ and $e_{2}$ be two edges of $X$ such that $i\left(e_{1}\right)=i\left(e_{2}\right)$ and the maximal common segment of the paths $\tilde{f}\left(e_{1}\right)$ and $\tilde{f}\left(e_{2}\right)$ is not a vertex. In this case we say that we have a partial fold. By subdividing appropriately the edges $e_{1}$ and

\footnotetext{
${ }^{1}$ It suffices to take $l^{\prime}=\frac{\|l\|_{1}}{1+l_{N}^{\prime}(e)} l_{N}^{\prime}$, where $l_{N}^{\prime}$ denotes the normalized Perron-Frobenius eigenvector of $M_{i}\left(\tilde{f}^{\prime}\right)$.
} 
$e_{2}$ as $e_{1}=e_{1}^{1} \circ e_{1}^{2}$ and $e_{2}=e_{2}^{1} \circ e_{2}^{2}$, we take the fold $\left(e_{1}^{1}, e_{2}^{1}\right)$ in such a way that the maximal common segment of the paths $\tilde{f}\left(e_{1}^{2}\right)$ and $\tilde{f}\left(e_{2}^{2}\right)$ is a vertex.

\subsection{Valence two homotopy.}

Definitions 3.27. Let $u$ be a degenerate vertex of $X$ such that the vertex $[u]$ in the quotient graph $X / G$ has valence two, provided that the two orbits of geometric edges are distinct. Then the star of $u$ (and of each vertex in the orbit $O_{u}$ ) consists exactly of two distinct edges $e_{1}$ and $e_{2}$ such that $t\left(e_{1}\right)=t\left(e_{2}\right)=u$. Without loss of generality, we can suppose that $e_{1} \in H_{i}$ and $e_{2} \in H_{j}$ with $i \leq j$. If $i=j$ and $H_{i}$ is exponentially growing, we suppose further that $w_{M_{i}(\tilde{f})}\left(e_{1}\right) \geq w_{M_{i}(\tilde{f})}\left(e_{2}\right)$, where $w_{M_{i}(\tilde{f})}$ denotes the unique normalized Perron-Frobenius left eigenvector of $M_{i}(\tilde{f})$ and $w_{M_{i}(\tilde{f})}\left(e_{i}\right)$ is the coordinate corresponding to $O_{e_{i}}$.

Let $X^{\prime}$ be the tree obtained from $X$ by collapsing the orbit $O_{e_{1}}$. We already know from Subsection 3.1 that $X^{\prime}$ is a $G$-tree, the natural projection $\pi: X \longrightarrow X^{\prime}$ is a $G$-map and the stabilizer of the vertex $\pi(u)=\pi\left(t\left(e_{1}\right)\right)$ is $G_{i\left(e_{1}\right)}$. We can view the groupoid $\pi X^{\prime}$ as a subgroupoid of $\pi X$ via the map $j: \pi X^{\prime} \longrightarrow \pi X$ defined by $j\left(\pi\left(g e_{2}\right)\right)=g e_{2} \circ g e_{1}^{-1}$. Note that $\pi$ is a bijection on $X \backslash O_{e_{1}}$ and that $j$ is a well-defined $G$-map since the star of $g u$ contains only the edge $g e_{1}$ from the orbit $O_{e_{1}}$. Also, reduced paths are mapped via $\pi$ on reduced paths.

In Subsection 3.1 the degeneration was enough in order to define the map $\tilde{f}^{\prime}$. Now the $G$-map $j$ is playing an important role. We note that $j$ is a section of $\left.\pi\right|_{X \backslash\left\{O_{e_{1}}, O_{e_{2}}\right\}}$. We define $\tilde{f}^{\prime}(p)=[\pi \circ \tilde{f} \circ j(p)]$ and $X_{i}^{\prime}=\pi\left(X_{i}\right)$. It is easy to see that $\tilde{f}^{\prime}$ is a representative of $f$ and that the $X_{i}^{\prime}$ 's are $\tilde{f}^{\prime}$-invariant subgraphs of $X^{\prime}$ that determine a filtration for $\left(\tilde{f}^{\prime}, X^{\prime}\right)$. In this case we say that $\left(\tilde{f}^{\prime}, X^{\prime}\right)$ is obtained from $(\tilde{f}, X)$ by a valence two homotopy along $e_{1}$.

Proposition 3.28. Suppose that $X$ has at least two orbits of vertices and that $\left(\tilde{f}^{\prime}, X^{\prime}\right)$ is obtained from $(\tilde{f}, X)$ by a valence two homotopy along $e_{1}$ as above.

(1) If $H_{i}$ is not exponentially growing, then $\Lambda=\Lambda^{\prime}$.

(2) If $i<j$ and $H_{i}$ is exponentially growing, then $\Lambda^{\prime}<\Lambda$.

(3) If $i=j$ and $H_{i}$ is exponentially growing, then from $H_{i}$ we obtain strata $H_{i 1}, \ldots, H_{i n}$ such that their matrices have Perron-Frobenius eigenvalues $\lambda_{i j}$ for which $\lambda_{i j} \leq \operatorname{PF}\left(M_{i}(\tilde{f})\right)$ for $j=1, \ldots, n$.

Proof. It is clear that valence two homotopy has no effect at strata $H_{l}$ for $l<i$. Let $H_{l}$ be an exponentially growing stratum with $l>i, l \neq j$ and $e \in H_{l}$. The effect of $\pi$ on $\tilde{f}(j(e))$ is to remove edges of the form $g e_{1}^{ \pm 1}$ for $g \in G$. Since $\tilde{f}^{\prime}(e)=[\pi \circ \tilde{f}(j(e))]$ and $l>i$, it follows that $M_{l}(\tilde{f})=M_{l}\left(\tilde{f}^{\prime}\right)$.

Suppose now that $i<j$ and that $e$ is an edge of $H_{j}$. If $e \notin O_{e_{2}}$, then the argument in the previous case works as well for $\tilde{f}^{\prime}(e)$. If $e \in O_{e_{2}}$, then $\tilde{f}^{\prime}\left(g e_{2}\right)=$ $\pi \tilde{f}\left(g e_{2} \circ g e_{1}^{-1}\right)=\pi\left(\tilde{f}\left(g e_{2}\right) \cdot \tilde{f}\left(g e_{1}^{-1}\right)\right)$. The cancelations on $\tilde{f}\left(g e_{2}\right) \cdot \tilde{f}\left(g e_{1}\right)^{-1}$ take place on $\pi X_{i}$ while $\pi$ does not collapse any edge of the stratum $H_{j}$. Thus we have $M_{j}(\tilde{f})=M_{j}\left(\tilde{f}^{\prime}\right)$.

We now consider the effect on $H_{i}$. If $i<j$, then the valence two homotopy along $e_{1}$ is a valence-one homotopy over $X_{i}$, since $u$ is a valence-one vertex of $X_{i}$. It follows that $\Lambda^{\prime}<\Lambda$ if $H_{i}$ is exponentially growing and $\Lambda^{\prime}=\Lambda$ otherwise. Thus we have established (1) and (2). 
Finally, we consider the case that $i=j$. Let

$$
\begin{gathered}
M_{i}(\tilde{f})=\left[\begin{array}{ccc}
A & B & B^{\prime} \\
m_{1} & n_{1} & n_{1}^{\prime} \\
m_{2} & n_{2} & n_{2}^{\prime}
\end{array}\right], \quad N=\left[\begin{array}{cc}
A & B \\
m_{1}+m_{2} & n_{1}+n_{2}
\end{array}\right], \\
M_{i}(j)=\left[\begin{array}{lll}
I & 0 & 0 \\
0 & 1 & 1
\end{array}\right], \quad \text { and } \quad M_{i}(\pi)=\left[\begin{array}{ll}
I & 0 \\
0 & 1 \\
0 & 0
\end{array}\right],
\end{gathered}
$$

where the two last columns of $M_{i}(\tilde{f})$ correspond to the orbits $O_{e_{2}}$ and $O_{e_{1}}$, respectively, and the last row of $N$ corresponds to the orbit of $\pi\left(e_{2}\right)=e$. We observe that $N=M_{i}(j) M_{i}(\tilde{f}) M_{i}(\pi)$ and that $M_{i}\left(\tilde{f}^{\prime}\right) \leq \mathrm{N}$ with inequality in the last row only due to cancellations in $\tilde{f}\left(e_{2}\right) \cdot \tilde{f}\left(e_{1}^{-1}\right)$. If $v$ is the normalized Perron-Frobenius left eigenvector of $M_{i}(\tilde{f})$, then there is a decomposition $v=\left(v_{0}, v_{2}, v_{1}\right)$ as a row vector such that $w_{M_{i}(\tilde{f})}\left(e_{1}\right)=v_{1}$, and $w_{M_{i}(\tilde{f})}\left(e_{2}\right)=v_{2}$ with $v_{1} \geq v_{2}$. We set $w=v M_{i}(\pi)=\left(v_{0}, v_{2}\right)$. Then, we have $v M_{i}(\pi) M_{i}(j)=\left(v_{0}, v_{2}, v_{2}\right) \leq\left(v_{0}, v_{2}, v_{1}\right)=v$ and hence $w N=\left(v M_{i}(\pi)\right) M_{i}(j) M_{i}(\tilde{f}) M_{i}(\pi)=\left(v M_{i}(\pi) M_{i}(j)\right) M_{i}(\tilde{f}) M_{i}(\pi) \leq$ $v M_{i}(\tilde{f}) M_{i}(\pi)=\lambda v M_{i}(\pi)=\lambda w$, where $\lambda=P F\left(M_{i}(\tilde{f})\right)$. Since $M_{i}\left(\tilde{f}^{\prime}\right) \leq N$, from the stratum $H_{i}^{\prime}$ we get strata $H_{i j}$ such that their matrices $M_{i j}$ are obtained from $N$ by deleting and reducing some elements of $N$. Therefore each $M_{i j}$ is dominated by $N$ and Proposition II.1.7 in [10] implies that the Perron-Frobenius eigenvalues $\lambda_{i j}$ of $M_{i j}$ 's are as in the statement of the proposition.

3.6. The elementary operations preserve the complexity. For a representative $\tilde{f}_{0}: X_{0} \longrightarrow X_{0}$ of a symmetric automorphism $f$ we denote by $\left\langle\left(\tilde{f}_{0}, X_{0}\right)\right\rangle$ the set of the representatives of $f$ that are obtained from $\left(\tilde{f}_{0}, X_{0}\right)$ by a finite sequence of elementary operations.

We remind that for a graph of groups $(\mathcal{G}, Y)$ the number of the nondegenerate vertices of $Y$ is denoted by $V_{d}(Y)$. We close this section with the following proposition.

Proposition 3.29. Let $\left(\tilde{f}_{0}, X_{0}\right)$ be a representative of $f$ as above and $(\tilde{f}, X) \in$ $\left\langle\left(\tilde{f}_{0}, X_{0}\right)\right\rangle$. If $\varphi: X_{0} \longrightarrow X$ is the composition of elementary operations, then:

(1) The edge and nondegenerate vertex groups of the $G$-action on $X$ are as in $X_{0}$.

(2) $V_{d}\left(X_{0} / G\right)=V_{d}(X / G)$.

(3) $r\left(X_{0} / G\right)=r(X / G)$.

(4) $C\left(X_{0} / G\right)=C(X / G)$.

Proof. We write $\varphi=\pi_{k} \circ \cdots \circ \pi_{1}$, where each $\pi_{i}$ is an elementary operation and $k$ is the minimum positive integer with this property. The proposition follows easily by induction on $k$ and analysis of the elementary operations in this section.

\section{Existence OF STABLE RELATIVE TRAin TRACK REPRESENTATIVES}

Let $G$ be a group acting on a tree $X$ and $f$ a symmetric automorphism of $G$, in view of Hypotheses 3.1 and Remark 3.3. Our main goal in the present section is to prove that there exists a relative train track representative for $f$.

We start with Subsection 4.1 where we extend the notions of bounded and relative train tracks maps (see [2]) to representatives of symmetric automorphisms. 
4.1. Relative train track representatives. Let $\left(\tilde{f}_{0}, X_{0}\right)$ be a pair which represents the automorphism $f$ of $G$ as in the previous section. Since we assumed $X_{0} / G$ to be finite, it follows that for each representative $(\tilde{f}, X) \in\left\langle\left(\tilde{f}_{0}, X_{0}\right)\right\rangle$ of $f$ the quotient graph $X / G$ is finite as well. Suppose that $X / G$ has no degenerate vertices of valence one and two and that $n, m$ are the numbers of the vertices and edges of $X / G$, respectively.

We consider the quotient graph $X / G$. If $V_{i}$ denotes the number of the vertices of valence $i$ in $X / G$, then since $r(X / G)=m-n+1$ we have

$$
\begin{aligned}
2(r(X / G)-1) & =\sum_{i>0}(i-2) V_{i}=-V_{1}+V_{3}+2 V_{4}+\cdots \\
& =-2 V_{1}-V_{2}+\left(V_{1}+V_{2}+V_{3}+2 V_{4}+\cdots\right) \geq-2 V_{1}-V_{2}+n,
\end{aligned}
$$

and hence $n \leq 2 V_{1}+V_{2}+2 r(X / G)-2$. Note also that $2 V_{1}+V_{2} \leq 2 V_{d}\left(X_{0} / G\right)$. It follows that $m=r\left(X_{0} / G\right)+n-1 \leq r\left(X_{0} / G\right)+2 V_{d}\left(X_{0} / G\right)+2 r\left(X_{0} / G\right)-2-1=$ $2 V_{d}\left(X_{0} / G\right)+3 r\left(X_{0} / G\right)-3$, and thus the number of the edges of $X / G$ is bounded by a positive integer $L=L\left(G, V_{d}\left(X_{0} / G\right), r\left(X_{0} / G\right)\right)$. In the case where $X_{0} / G$ is a loop with a degenerate vertex, we set $L=1$. In this case one can check directly that the subgroup Fix $(f)$ has complexity at most 1 for any endomorphism $f$ of $G$.

Remark 4.1. From the Bass-Serre theory we know that there is an epimorphism $G \longrightarrow \pi_{1}(X / G)$. Thus, if $G$ is finitely generated, the rank $r(X / G)=r\left(X_{0} / G\right)$ of $\pi_{1}(X / G)$ is bounded by the minimum number of generators $d(G)$ of $G$ and the above inequality yields $m \leq 2 V_{d}\left(X_{0} / G\right)+3 d(G)-3$.

Definitions 4.2. Following [2], the representative $(\tilde{f}, X) \in\left\langle\left(\tilde{f}_{0}, X_{0}\right)\right\rangle$ is called bounded if it determines at most $L$ exponentially growing strata $H_{r}$ whose $\operatorname{PF}\left(M_{r}(\tilde{f})\right.$ 's are the Perron-Frobenius eigenvalues of matrices of size at most $L \times L$.

Let $k$ be a positive constant and $\mathcal{M}_{k}$ the set of the irreducible matrices of nonnegative integers of size at most $L \times L$ with Perron-Frobenius eigenvalue at most $k$. Proposition 2.3(iii) implies that the set $\mathcal{M}_{k}$ is finite and hence the set $\left\{P F(M), M \in \mathcal{M}_{k}\right\}$ is finite as well. From this we conclude that the set of the sequences $\Lambda(M(\tilde{f}))$ of the bounded representatives of $f$ is a discrete subset of $\mathbb{R}^{L}$ and therefore $\Lambda$ achieves a minimum value $\Lambda_{\text {min }}$ among all bounded representatives $\tilde{f}: X \longrightarrow X$. The representative $\tilde{f}$ is called minimal if $\Lambda(M(\tilde{f}))=\Lambda_{\min }$.

Let $\tilde{f}$ be a representative of $f$ and $\tilde{f}^{\prime}$ a representative obtained by $\tilde{f}$ by an elementary operation. We call the elementary operation safe if $\Lambda\left(M\left(\tilde{f}^{\prime}\right)\right) \leq \Lambda(M(\tilde{f}))$. We have already seen, in Section 3, that each elementary operation different to valence two homotopy is safe.

For the proof of the following fact we refer the reader to [2].

Lemma 4.3. Let $\tilde{f}: X \longrightarrow X$ be a bounded repersentative of $f$ from which we obtain $\tilde{f}^{\prime}: X^{\prime} \longrightarrow X^{\prime}$ by a sequence of safe elementary operations. If $\Lambda^{\prime}<\Lambda$, then there is a bounded representative $\tilde{f}^{\prime \prime}: X^{\prime \prime} \longrightarrow X^{\prime \prime}$ such that $\Lambda^{\prime \prime}<\Lambda$. In particular, if $\tilde{f}$ is minimal, then $\tilde{f}^{\prime}$ is minimal as well.

Since from any representative with valence one and two homotopies we find a bounded one, from now on we may assume that $\left(\tilde{f}_{0}, X_{0}\right)$ is bounded. Also, in view of Lemma 4.3 we restrict our attention to the set $\left\langle\left(\tilde{f}_{0}, X_{0}\right)\right\rangle_{s}$ which consists of those representatives in $\left\langle\left(\tilde{f}_{0}, X_{0}\right)\right\rangle$ that are obtained by a sequence of safe elementary operations. 
The analysis of the elementary operations in the previous section shows that each safe operation on a minimal representative gives a unique exponentially growing stratum $H_{r}^{\prime}$ for each exponentially growing stratum $H_{r}$ of $\tilde{f}$, since in this case $\operatorname{PF}\left(M_{r}(\tilde{f})\right)=\operatorname{PF}\left(M_{r}\left(\tilde{f}^{\prime}\right)\right)$. Note that, if $(\tilde{f}, X)$ is a minimal representative, then valence one homotopies and $\tilde{f}$-trivial edges do not occur in exponentially growing strata.

Definition 4.4. If $\tilde{f}: X \longrightarrow X$ is a representative of $f$, then we define the map $D \tilde{f}: E(X) \longrightarrow E(X)$ which maps an edge $e$ to the first edge of the geodesic $\tilde{f}(e)$ (because of the collapse we may assume that there are no $\tilde{f}$-trivial edges). A nontrivial turn $e_{1} \circ e_{2}$ is called illegal if for some exponent $k>0$ its image under of $(D \tilde{f})^{k}$ is a vertex (that is, $\left.\tilde{f}^{k}\right|_{e_{1} \circ e_{2}}$ is not an immersion), otherwise the turn $e_{1} \circ e_{2}$ is called legal. A path having no illegal turns in $H_{r}$ is called $r$-legal.

Remark 4.5. It is easy to see that $(D \tilde{f})^{k}(g e)=f^{k}(g)(D \tilde{f})^{k}(e)$, for each edge $e$ of $X$ and $g \in G$. Thus the property of being a turn illegal is preserved by the action of $G$.

Remark 4.6. From the above relation we see that the stabilizer of $(D \tilde{f})^{k}(e)$ contains $f^{k}\left(G_{e}\right)$, where $G_{e}$ denotes the stabilizer of $e$. Since all edge groups have the same cardinality, and $f$ is a monomorphism, it follows that the stabilizer of $(D \tilde{f})^{k}(e)$ is $f^{k}\left(G_{e}\right)$. Suppose now that $(D \tilde{f})^{k}(e)=(D \tilde{f})^{k}(g e)$ for some $g \in G$. Then $(D \tilde{f})^{k}(e)=f^{k}(g)(D \tilde{f})^{k}(e)$ and hence $f^{k}(g) \in f^{k}\left(G_{e}\right)$. We therefore get that $g \in G_{e}$. This shows that there are not any illegal turns of the form $e^{-1} \circ g e$.

We now recall, from [2], the definition of the relative train track map.

Definition 4.7. The representative $\tilde{f}: X \longrightarrow X$ is a relative train track map if the following conditions are satisfied for all exponentially growing strata $H_{r}$ :

RTT1. If $e$ is an edge in $H_{r}$, then the edges $D \tilde{f}(e)$ and $D \tilde{f}\left(e^{-1}\right)$ lie in $H_{r}$. In particular, each turn $e_{1} \circ e_{2}$ with $e_{1}$ in $H_{r}$ and $e_{2}$ in $X_{r-1}$ is legal.

RTT2. If $p \subseteq X_{r-1}$ is a nontrivial path with endpoints in $H_{r} \cap X_{r-1}$, then $\tilde{f}(p)$ is a nontrivial path with endpoints in $H_{r} \cap X_{r-1}$.

RTT3. If $p \subseteq H_{r}$ is a legal path, then the path $\tilde{f}(p)$ is r-legal.

The following lemma follows easily from the definition.

Lemma 4.8. Let $\tilde{f}: X \longrightarrow X$ be a relative train track map and let $p \subseteq X_{r}$ be an r-legal path. Then $\tilde{f}(p)$ is r-legal.

For the proof of the following lemma we refer the reader to [2].

Lemma 4.9. If $\tilde{f}: X \longrightarrow X$ is a minimal representative of $f$ in $\left\langle\left(\tilde{f}_{0}, X_{0}\right)\right\rangle$ satisfying RTT1, then $\tilde{f}$ satisfies RTT3. In particular, $\tilde{f}$ maps every edge of an exponentially growing stratum $H_{r}$ to an r-legal path.

4.2. Length functions on paths and the bounded cancellation lemma for $G$-trees. Let $(\tilde{f}, X)$ be a representative of $f$ and let $H_{r}$ be an exponentially growing stratum. In this subsection, following closely the approach in [10, we first set the length of each edge $e \in H_{r}$ to be equal to the entry of a Perron-Frobenius eigenvector corresponding to its orbit, and show that in the case where $\tilde{f}$ is a relative train track map, $\tilde{f}$ expands each r-legal path in $X_{r}$ by a factor of $\lambda_{r}=P F\left(M_{r}(\tilde{f})\right)$. Then, we 
prove the bounded cancellation lemma in our setting which is used to control the cancellation that takes place in the product $\tilde{f}\left(p_{1}\right) \cdot \tilde{f}\left(p_{2}\right)$, where $p_{1}$ and $p_{2}$ are the two r-legal parts of an indivisible Nielsen path in $X_{r}$ containing edges of $H_{r}$.

Definitions 4.10. If $p \in \pi X$ we denote by $[p]_{X}$ the nonnegative integral row vector $1 \times n$, whose $i$-coordinate is the number of times the path $p$ crosses the orbit $O_{i}$, regardless of orientation. If $p$ is a vertex then $[p]_{X}=\overrightarrow{0}$.

Each nonzero, nonnegative, real column vector $n \times 1, l$ defines a length function on $\pi X$ (Dicks-Ventura call this pseudometric) $L: \pi X \longrightarrow[0, \infty)$ by the rule $L(p)=$ $[p]_{X} \cdot l$. The length of the orbit $O_{i}$ is the $i$-coordinate $l(i)$ of $l$. The volume $\operatorname{vol}(X, l)=\operatorname{vol}(X)$ is the sum of $l(i)$ 's for $i=1, \ldots, n$. Thus $\operatorname{vol}(X)=\sum_{i=1}^{n} l(i)=$ $\|l\|_{1}$. We will say that $l$ is normalized if $\|l\|_{1}=1$.

Remarks 4.11. If $p_{1}$ and $p_{2} \in \pi X$, then $L\left(p_{1} \cdot p_{2}\right) \leq L\left(p_{1}\right)+L\left(p_{2}\right)$. Equality holds if the product $p_{1} \cdot p_{2}$ is cancellation free. Also, $[g p]_{X}=[p]$ and $l(g p)=l(p)$ for any $p \in \pi X$ and $g \in G$. Note that the i-row of the matrix $M(\tilde{f})$ is $\left[\tilde{f}\left(e_{i}\right)\right]_{X}=$ $\left[e_{i}\right]_{X} \cdot M(\tilde{f})$, for $e_{i} \in O_{i}$. In general we have $[\tilde{f}(p)]_{X} \leq[p]_{X} \cdot M(\tilde{f})$.

Proposition 4.12. Let $\tilde{f}: X \longrightarrow X$ be a relative train track map and $H_{r}$ an exponentially growing stratum. If $\lambda_{r}=P F\left(M_{r}(\tilde{f})\right)$, then there exists a length function $L$ on $\pi X$ such that $L_{r}(\tilde{f}(p)) \leq \lambda_{r} L_{r}(p)$ for every path $p$ in $X_{r}$; moreover $L_{r}(\tilde{f}(p))=\lambda_{r} L_{r}(p)$ for every r-legal path $p$ in $X_{r}$.

Proof. We define $L_{r}(p)=[p]_{H_{r}} \cdot l_{r}$ for any path in $\pi X$, where $l_{r}$ is a right eigenvector of the transition matrix $M_{r}(\tilde{f})$. We observe that all paths in $X_{r-1}$ have length zero, and that each non-trivial path containing an edge of $H_{r}$ has strictly positive length in view of the positivity of $l_{r}$. The fact that $l_{r}$ is a right eigenvector of $M_{r}$ and the relation $[\tilde{f}(e)]_{H_{r}}=[e]_{H_{r}} \cdot M_{r}(\tilde{f})$ imply that $L_{r}(\tilde{f}(e))=\lambda_{r} L_{r}(e)$ for every edge $e$ of $H_{r}$.

If now $p=e_{i_{1}} \circ \cdots \circ e_{i_{n}}, e_{i_{j}} \in E(X)$ is a path in $X_{r}$, then $L_{r}(\tilde{f}(p))=[\tilde{f}(p)]_{H_{r}} \cdot l_{r} \leq$ $\left(\sum_{j=1}^{n}\left[\tilde{f}\left(e_{i_{j}}\right)\right]_{H_{r}}\right) \cdot l_{r}=\sum_{e_{i_{j}} \in H_{r}}\left[\tilde{f}\left(e_{i_{j}}\right)\right]_{H_{r}} \cdot l_{r}=\sum_{e_{i_{j}} \in H_{r}} L_{r}\left(\tilde{f}\left(e_{i_{j}}\right)\right)=\sum_{e_{i_{j}} \in H_{r}} \lambda_{r}$. $L_{r}\left(e_{i_{j}}\right)=\lambda_{r} \sum_{e_{i_{j}} \in H_{r}} L_{r}\left(e_{i_{j}}\right)=\lambda_{r} L_{r}(p)$. In the case where the path $p$ is r-legal in the first inequality above we have equality, and this observation completes the proof.

The first inequality in the above proof is equality if and only if for each turn $e_{1} \circ e_{2}$ of $p$ in $H_{r},\left.\tilde{f}\right|_{e_{1} \circ e_{2}}$ is an immersion. Thus, by Lemma 4.8 and induction on $n$ we get the following corollary.

Corollary 4.13. Under the hypotheses of the above proposition the path $p$ is $r$-legal if and only if $L_{r}\left(\tilde{f}^{n}(p)\right)=\lambda_{r}^{n} L_{r}(p)$ for every $n$.

At this point we state the following proposition whose proof makes use of the last assertion of Proposition 4.12 and is exactly as the proof of Proposition IV.3.2 in [10].

Proposition 4.14. Suppose that $\tilde{f}: X \longrightarrow X$ is a minimal relative train track representative of $f$ which has no fixed points not vertices and that $H_{r}$ is an exponentially growing stratum. If $p \subseteq X_{r}$ is an indivisible Nielsen path intersecting $H_{r}$, then it contains a unique illegal turn in $H_{r}$. Moreover, if $p=p_{1} \circ p_{2}$ is the decomposition of $p$ into r-legal subpaths, then $\tilde{f}\left(p_{1}\right)=p_{1} \circ t$ and $\tilde{f}\left(p_{2}\right)=t^{-1} \circ p_{2}$ for some nontrivial r-legal path $t$. 
Corollary 4.15. Under the hypotheses of the above proposition, the first and last edges of the paths $p, p_{1}$ and $p_{2}$ lie in $H_{r}$.

Proof. We write $p_{1}=a \circ p^{\prime}$ with $a \subseteq X_{r-1}$ such that the first edge $e$ of $p^{\prime}$ lies in $H_{r}$. The last edge of $p_{1}$ is in $H_{r}$, being the first edge of the unique illegal turn of $p$. It follows that $p^{\prime}$ is not contained in $X_{r-1}$ and is not a vertex; also it is r-legal because $p_{1}$ is. By Property RTT1 the first and last edges of $\tilde{f}(e)$ lie in $H_{r}$ while $p^{\prime}$ is r-legal. Thus the first and last edges of $\tilde{f}\left(p^{\prime}\right)$ lie in $H_{r}$ as well and $\tilde{f}\left(a \circ p^{\prime}\right)=\tilde{f}(a) \circ \tilde{f}\left(p^{\prime}\right)=p_{1} \circ t=a \circ p^{\prime} \circ t$. Now, from the inclusion $\tilde{f}(a) \subseteq X_{r-1}$, we conclude that $\tilde{f}(a)=a$ and $\tilde{f}\left(p^{\prime}\right)=p^{\prime} \circ t$. But $p$ is an indivisible Nielsen path; therefore $a$ must be trivial. The same arguments show that the last edge of $p_{1}$ lies in $H_{r}$. Similarly for $p_{2}$.

Definitions 4.16. Let $X$ and $X^{\prime}$ be $G$-graphs whose each component is a tree, with length functions $L$ and $L^{\prime}$, respectively, and let $\varphi: \pi X \longrightarrow \pi X^{\prime}$ be a $\phi$-equivariant morphism of groupoids, that is, $\varphi(g p)=\phi(g) \varphi(p), \phi \in \operatorname{End}(G)$. The number given by

$$
\operatorname{Lip}(\varphi)=\sup \left\{\frac{L^{\prime}(\varphi(e))}{L(e)}, e \in E(X), L(e) \neq 0\right\}
$$

is called the Lipschitz number of $\varphi$ and the number

$$
C B(\varphi)=\frac{1}{2} \sup \left\{L^{\prime}(\varphi(p))+L^{\prime}(\varphi(q))-L^{\prime}(\varphi(p \circ q)), p, q \in \pi X\right\}
$$

is called the cancellation bound of $\varphi$. A morphism $\varphi$ preserves length functions if $L^{\prime}(\varphi(e))=L(e)$ for all $e \in E(X)$.

Suppose that $L(e)=0$ implies $L^{\prime}(\varphi(e))=0$, for all $e \in E(X)$. Then it is clear that $L^{\prime}(\varphi(p)) \leq \operatorname{Lip}(\varphi) L(p)$. Let $\tilde{f}_{r}: X_{r} \longrightarrow X_{r}$ be the restriction of a representative of $f$ on the stratum $X_{r}$ and let $L_{r}$ be the length function that is defined by a right eigenvector of the transition matrix $M_{r}(\tilde{f})$. We observe that if $L_{r}(e)=0$, then $L_{r}\left(\tilde{f}_{r}(e)\right)=0$.

We have already seen that the representative $\tilde{f}$ does not admit partial fold in the same orbit. Hence $\tilde{f}_{r}$ can be expressed as a (finite) composition $\tilde{f}_{r}=\varphi \circ \varphi_{k} \circ$ $\cdots \circ \varphi_{1} \circ \varphi_{0}$, where each $\varphi_{i}: X_{r}^{i-1} \longrightarrow X_{r}^{i}$ for $i=1, \ldots, k$ is a fold, $\varphi_{0}: X_{r} \longrightarrow X_{r}^{0}$ colapses $\tilde{f}$-trivial edges and subdivide, and $\varphi: X_{r}^{m} \longrightarrow X_{r}$ is locally injective. In particular, $\varphi$ and $\varphi_{i}$ for $i=1, \ldots, k$ are maps of graphs.

We equip each $X_{r}^{i}$ for $i=0, \ldots, k$ with the length function induced from $X_{r}$; therefore the fold maps and $\varphi$ preserve length functions, and from the above definitions we see immediately that $\operatorname{Vol}\left(X_{r}^{0}\right) \leq \operatorname{Lip}\left(\tilde{f}_{r}\right) \cdot \operatorname{Vol}\left(X_{r}\right)$. Also, we note that $\operatorname{Lip}(\varphi)=\operatorname{Lip}\left(\varphi_{i}\right)=1$ for $i=1, \ldots, k, C B\left(\varphi_{0}\right)=C B(\varphi)=0$, and that $C B\left(\varphi_{i}\right)=\operatorname{Vol}\left(X_{r}^{i-1}\right)-\operatorname{Vol}\left(X_{r}^{i}\right)=$ the length of the image of the corresponding fold. Now, it is not hard to see that $C B\left(\varphi_{i+1} \varphi_{i}\right) \leq C B\left(\varphi_{i+1}\right)+\operatorname{Lip}\left(\varphi_{i+1}\right) C B\left(\varphi_{i}\right)$ for $i=0, \ldots, k$, where $\varphi_{k+1}=\varphi$. Thus we have $C B\left(\tilde{f}_{r}\right)=C B\left(\varphi \circ \varphi_{k} \circ \cdots \circ \varphi_{1} \circ \varphi_{0}\right) \leq$ $C B(\varphi)+\operatorname{Lip}(\varphi) C B\left(\varphi_{k} \circ \cdots \circ \varphi_{1} \circ \varphi_{0}\right)=C B\left(\varphi_{k} \circ \cdots \circ \varphi_{1} \circ \varphi_{0}\right) \leq \sum_{i=1}^{k} C B\left(\varphi_{i}\right)=$ $\sum_{i=1}^{k}\left(\operatorname{Vol}\left(X_{r}^{i-1}\right)-\operatorname{Vol}\left(X_{r}^{i}\right)\right)=\operatorname{Vol}\left(X_{r}^{0}\right)-\operatorname{Vol}\left(X_{r}^{k}\right) \leq \operatorname{Vol}\left(X_{r}^{0}\right) \leq \operatorname{Lip}\left(\tilde{f}_{r}\right) \cdot \operatorname{Vol}\left(X_{r}\right)$, and the following lemma is proved.

Lemma 4.17. If $\tilde{f}: X \longrightarrow X$ is a representative of $f$, and $L_{r}$ is the length function defined by a Perron-Frobenius right eigenvector $l_{r}$ of the matrix $M_{r}(\tilde{f})$ as above, then $C B\left(\tilde{f}_{r}\right) \leq \operatorname{Lip}\left(\tilde{f}_{r}\right)\left\|l_{r}\right\|_{1}$. 
Corollary 4.18. Let $\tilde{f}: X \longrightarrow X$ be a minimal relative train track representative of $f$ which has no fixed points not vertices, $H_{r}$ an exponentially growing stratum, $l_{r}$ a Perron-Frobenius right eigenvector of the transition matrix $M_{r}$ and $L_{r}$ the corresponding length function. If $p \subseteq X_{r}$ is an indivisible Nielsen path that intersects $H_{r}$, then $L_{r}\left(p_{1}\right)=L_{r}\left(p_{2}\right) \leq \frac{\lambda_{r}}{\lambda_{r}-1}\left\|l_{r}\right\|_{1}$, where $\lambda_{r}=P F\left(M_{r}(\tilde{f})\right)$, and $p_{1}, p_{2}$ are the two r-legal parts of $p$.

Proof. By Proposition 4.14, we know that $p=p_{1} \circ p_{2}$, where $p_{1}$ and $p_{2}$ are r-legal paths with $\tilde{f}\left(p_{1}\right)=p_{1}$ ot and $\tilde{f}\left(p_{2}\right)=t^{-1} \circ p_{2}$. From this we conclude that $C B\left(\tilde{f}_{r}\right) \geq$ $L_{r}(t)$. On the other hand, by the above lemma we have $C B\left(\tilde{f}_{r}\right) \leq \operatorname{Lip}\left(\tilde{f}_{r}\right)\left\|l_{r}\right\|_{1}$. Thus $L_{r}(t) \leq \lambda_{r}\left\|l_{r}\right\|_{1}$ since $\operatorname{Lip}\left(\tilde{f}_{r}\right)=\lambda_{r}$. Now, by Proposition $4.12 \lambda_{r} L_{r}\left(p_{i}\right)=$ $L_{r}\left(\tilde{f}\left(p_{i}\right)\right)=L_{r}\left(p_{i}\right)+L_{r}(t)$ for $i=1,2$, and hence $L_{r}\left(p_{i}\right)=\frac{L_{r}(t)}{\lambda_{r}-1} \leq \frac{\lambda_{r}}{\lambda_{r}-1}\left\|l_{r}\right\|_{1}$.

\subsection{Stable representatives.}

Definitions 4.19. Under the hypotheses of Corollary 4.18, for each indivisible Nielsen path $p$ which intersects the stratum $H_{r}$, there is a uniform bound $m$ on the number of the edges $e_{i_{1}}, \ldots, e_{i_{n}}, n \leq m$ (the order of these comes from the series of appearance) in $p \cap H_{r}$. So, each indivisible Nielsen path $p$ as above defines an element $\left(x_{\lambda}\right)_{\lambda}$ of $A^{2 n+1}$, where $A$ is the set $\left\{O_{y_{1}}^{ \pm 1}, \ldots, O_{y_{k}}^{ \pm 1}, 0,1: H_{r}=\bigsqcup_{i=1}^{k} O_{y_{i}}\right\}$, such that:

(1) $x_{2 \lambda-1}$ is the orbit of the edge $e_{i_{\lambda}}$, and

(2) $x_{2 \lambda}=0$ or 1 with $x_{2 \lambda}=0$ if and only if the edges $e_{i_{\lambda}}$ and $e_{i_{\lambda+1}}$ are successive in $p$ of the form $g x$ and $g y$, respectively, where $x$ and $y$ are edges of $X$.

We define $N(\tilde{f}, r)$ to be the number of these elements for paths $p \subseteq X_{r}$ that intersect $H_{r}$. It is clear that $N(\tilde{f}, r)<\infty$. If we set $N(\tilde{f})=\sum N(\tilde{f}, r)$, where the sum is taken on the exponentially growing strata, we have $N(\tilde{f})<\infty$ and define $N_{\text {min }}$ to be the minimum value of $N(\tilde{f})$ among all $\tilde{f} \in\left\langle\left(\tilde{f}_{0}, X_{0}\right)\right\rangle_{s}$ with $\operatorname{FPnV}(\tilde{f})=0$, where $\left(\tilde{f}_{0}, X_{0}\right)$ is a minimal bounded representative of $f$. The representative $\tilde{f}: X \longrightarrow X$ is called stable if it is obtained from a minimal bounded by a sequence of safe operations (hence $\Lambda(\tilde{f})=\Lambda_{\text {min }}$ ), $\operatorname{FPnV}(\tilde{f})=0$ and $N(\tilde{f})=N_{\text {min }}$.

For the existence of relative train track representatives $\tilde{f}$ with $\Lambda(\tilde{f})=\Lambda_{\min }$ and $\operatorname{FPnV}(\tilde{f})=0$, in order to have $N_{\min }<\infty$, see the remark after Theorem 4.22.

The definition of stability is more complicated than this one in 2. The stability is defined in such a way to be preserved by subdivisions, core subdivisions and folds. The proof of them is left as an exersise to the reader.

For later use, we prove at this point the following proposition.

Proposition 4.20. Let $\tilde{f}: X \longrightarrow X$ be a stable relative train track representative of $f$ and $H_{r}$ an exponentially growing stratum. Then $H_{r}$ does not contain indivisible Nielsen paths of the form $p=e_{1}^{-1} \circ e_{2}$.

Proof. Suppose the contrary and let $p=e_{1}^{-1} \circ e_{2}$ be an indivisible Nielsen path where $e_{1}$ and $e_{2}$ are edges of $H_{r}$. From Proposition 4.14 we have $\tilde{f}\left(e_{1}\right)=t \circ e_{1}$ and $\tilde{f}\left(e_{2}\right)=t \circ e_{2}$. We first subdivide each $e_{i}$ for $i=1,2$ as $e_{i}=e_{i}^{1} \circ e_{i}^{2}$ such that $\tilde{f}\left(e_{i}^{1}\right)=t$ and $\tilde{f}\left(e_{i}^{2}\right)=e_{i}=e_{i}^{1} \circ e_{i}^{2}$. Then we fold the edges $e_{1}^{1}$ and $e_{2}^{1}$ and again subdivide $e_{i}^{2}=e_{i}^{21} \circ e_{i}^{22}$ such that $\tilde{f}\left(e_{i}^{21}\right)=e_{1}^{1}=e_{2}^{1}$ and $\tilde{f}\left(e_{i}^{22}\right)=e_{i}^{2}=e_{i}^{21} \circ e_{i}^{22}$. Finally, we fold the edges $e_{1}^{21}$ and $e_{2}^{21}$. 

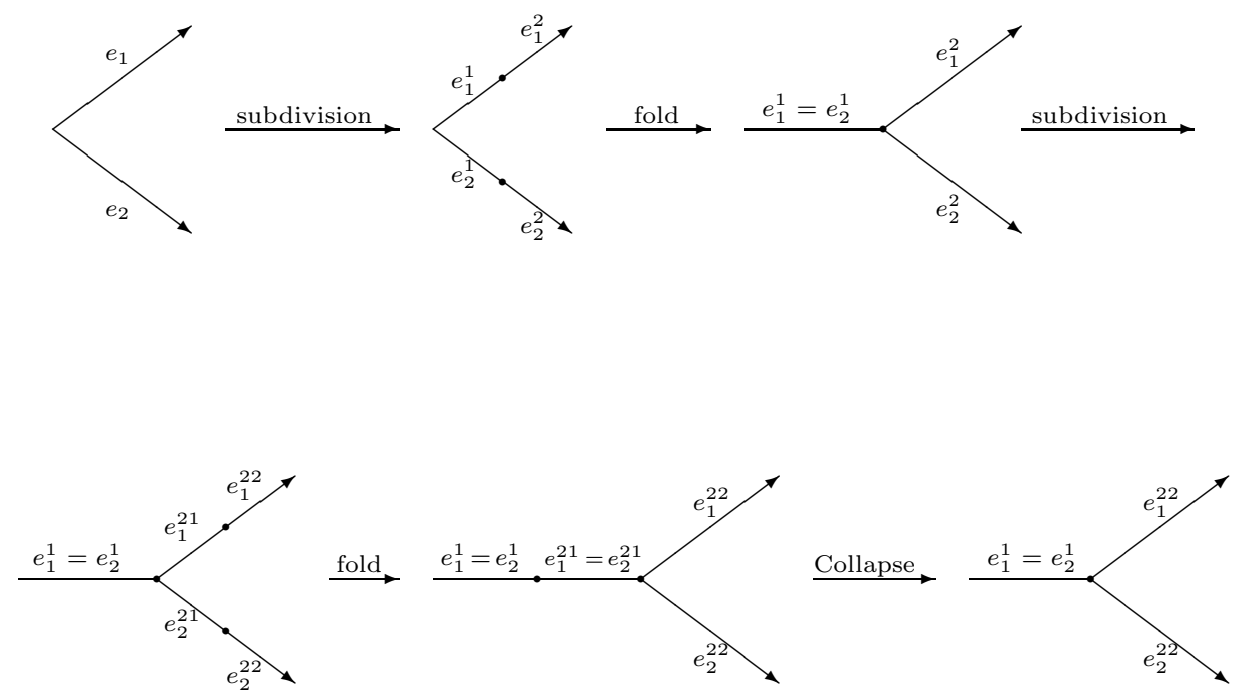

If the matrix $M_{r}(\tilde{f})$ has the form

$$
\left[\begin{array}{ccc}
A & C_{1} & C_{2} \\
L & m+1 & n \\
L & m & n+1
\end{array}\right]
$$

then since the representative $\tilde{f}$ is minimal, the corresponding matrix after these operations will be

$$
M_{r}=\left[\begin{array}{ccccc}
A & C_{1}+C_{2} & C_{1}+C_{2} & C_{1} & C_{2} \\
L & m+n & m+n & m & n \\
0 & 1 & 0 & 0 & 0 \\
0 & 0 & 1 & 1 & 0 \\
0 & 0 & 1 & 0 & 1
\end{array}\right] .
$$

Now, by collapsing the orbit of the edge $e_{1}^{21}$ we obtain the matrix

$$
N^{\prime}=\left[\begin{array}{cccc}
A & C_{1}+C_{2} & C_{1} & C_{2} \\
L & m+n+1 & m & n \\
0 & 0 & 1 & 0 \\
0 & 0 & 0 & 1
\end{array}\right] .
$$

Obviously $N^{\prime}$ is not irreducible, but it follows as in the proof of Lemma 3.8 in [2] that the submatrix $N=\left[\begin{array}{cc}A & C_{1}+C_{2} \\ L & m+n+1\end{array}\right]$ of $N^{\prime}$ that is obtained from $N^{\prime}$ by deleting the rows and columns corresponding to $O_{e_{1}^{22}}$ and $O_{E_{2}^{22}}$ is irreducible; therefore $N$ defines a maximal stratum while the orbits $O_{e_{1}^{22}}$ and $O_{e_{2}^{22}}$ constitute nonexponentially growing strata with $\lambda=1$.

Let

$$
\vec{v}=\left[\begin{array}{c}
\overrightarrow{v_{0}} \\
v_{1} \\
v_{2} \\
v_{3} \\
v_{4}
\end{array}\right]
$$


be a right Perron-Frobenius eigenvector of $M_{r}$ and let $\lambda_{r}$ be the corresponding eigenvalue. If we define $w$ to be the column vector $\left[\begin{array}{c}\overrightarrow{v_{0}} \\ v_{1}+v_{2}+v_{3}\end{array}\right]$, then $N w=\lambda_{r} w$ and Proposition 2.3 implies that this collapse is safe. Now, it is easy to check that this collapse does not create fixed points not vertices and that the number $N(\tilde{f})=N_{\text {min }}$ has decreased by at least one which gives a contradiction.

We now recall the following lemma from [2], whose proof in our case requires only subdivisions and folds which take place in $X_{i-1}$.

Lemma 4.21. Suppose that $\tilde{f}: X \longrightarrow X$ is a stable representative of $f$, and that $p \subseteq X_{i-1}$ is a path with endpoints in $H_{i} \cap X_{i-1}$ such that $\tilde{f}(p)$ is a vertex. Then there exists a stable representative $\tilde{f}^{\prime}: X^{\prime} \longrightarrow X^{\prime}$ of $f$ such that $H_{i}^{\prime} \cap X_{i-1}^{\prime}$ has fewer vertices than $H_{i} \cap X_{i-1}$. Moreover, if $j \geq i$ and $H_{j}$ satisfies RTT1, then $H_{j}^{\prime}$ satisfies RTT1. If $j>i$ and $H_{j}$ satisfies RTT1 and RTT2, then $H_{j}^{\prime}$ satisfies RTT1 and RTT2.

We are now ready to prove the main result of this section.

Theorem 4.22 ([2, Theorem 5.12]). For every summetric automorphism $f$ of $G$ there exists a stable relative train track representative.

Proof. We have already seen that we can assume that all elementary operations take place over $\left\langle\left(\tilde{f}_{0}, X_{0}\right)\right\rangle_{s}$, where $\tilde{f}_{0}$ is a bounded minimal representative of $f$. Also, without loss of generality, we may assume moreover that $\operatorname{FPnV}\left(\tilde{f}_{0}\right)=0$ (otherwise with subdivisions at the fixed points which are not vertices we find a minimal representative $\left(\tilde{f}_{1}, X_{1}\right) \in\left\langle\left(\tilde{f}_{0}, X_{0}\right)\right\rangle_{s}$ with $\operatorname{FPnV}\left(\tilde{f}_{1}\right)=0$ and work on $\left.\left\langle\left(\tilde{f}_{1}, X_{1}\right)\right\rangle_{s} \subseteq\left\langle\left(\tilde{f}_{0}, X_{0}\right)\right\rangle_{s}\right)$.

We choose over $\left\langle\left(\tilde{f}_{0}, X_{0}\right)\right\rangle_{s}$ a stable representative of $f$ and perform core subdivisions until the top exponentially growing stratum satisfies property RTT1. We recall that each core subdivision on a stratum $H_{r}$ preserves stability since $F \operatorname{Pn} V\left(\tilde{f}_{0}\right)=0$ as well as property RTT1 for strata $H_{l}$ with $l \neq r$, because it has no effect on a different exponentially growing stratum. Then we continue applying Lemma 4.21, starting from the top stratum in order to take a stable representative $(\tilde{f}, X)$ satisfying property RTT2 on it. Repeating this procedure in the remaining exponentially growing strata we obtain a stable representative $(\tilde{f}, X)$ satisfying properties RTT1 and RTT2 on each exponentially growing stratum. Property RTT3 is now satisfied because of Lemma 4.9.

Remark 4.23. The proof of the above theorem shows in particular the existence of relative train track representatives in $\left\langle\left(\tilde{f}_{0}, X_{0}\right)\right\rangle_{s}$ and this ensures that $N_{\min }<\infty$.

\section{Folding at the illegal turn of Indivisible Nielsen paths}

Let $\tilde{f}: X \longrightarrow X$ be a stable relative train track representative of the automorphism $f$ and let $H_{r}$ be an exponentially growing stratum. The purpose of this section is to prove that all indivisible Nielsen paths that intersect $H_{r}$ and are contained in $X_{r}$ belong to the same orbit under the action of $G$.

We start with the description of a series of elementary operations involving an indivisible Nielsen path $p$. The result is that we call folding over $p$.

By Proposition 4.14, the path $p$ has a decomposition $p=p_{1} \circ p_{2}$, where each $p_{i}$ is r-legal for $i=1,2$ and whose unique illegal turn in $H_{r}$ is $e_{1}^{-1} \circ e_{2}$. If $p_{1}=s \circ e_{1}^{-1}$, then since $p_{1}$ is r-legal we have $s \circ e_{1}^{-1} \circ t=p_{1} \circ t=\tilde{f}\left(p_{1}\right)=\tilde{f}(s) \circ \tilde{f}\left(e_{1}^{-1}\right)$ while 
$\tilde{f}$ being stable has no fixed points which are not vertices. It follows that either $\tilde{f}\left(e_{1}\right)=t^{-1} \circ e_{1}$ (in this case $e_{1}^{-1}$ is the first edge of $p$ ) or the path $\tilde{f}\left(e_{1}\right)$ is an initial segment of $t^{-1}$. Similarly, we see that either $\tilde{f}\left(e_{2}\right)=t^{-1} \circ e_{2}$ (and $e_{2}$ is the last edge of $p$ ) or $\tilde{f}\left(e_{2}\right)$ is an initial segment of $t^{-1}$. Thus, because of Proposition 4.20 without loss of generality we may assume that $\tilde{f}\left(e_{2}\right)$ is an initial segment of $t^{-1}$ and of $\tilde{f}\left(e_{1}\right)$. Let $l_{r}$ be a right eigenvector of the transition matrix $M_{r}$ and $L_{r}$ the length function on $\pi X$ induced by $l_{r}$. We distinguish two cases.

Case 1: $\tilde{f}\left(e_{1}\right)=\tilde{f}\left(e_{2}\right)$. Then perform the fold $\left(e_{1}, e_{2}\right)$ and if the new stable representative is a relative train track map we stop. If not, then continuing as in the proof of Theorem 4.22 we get a stable relative train track representative. In any case we have reduced the number of the edges of $H_{r}$ by one since the core subdivision does not increase $H_{r}$ and the other operations described in the proof of Theorem 4.22 take place in $X_{i}$ for $i<r$.

Case 2: $\tilde{f}\left(e_{1}\right)>\tilde{f}\left(e_{2}\right)$. Then, there are two subcases depending on whether the next edge of $e_{2}$ in $p$ belongs to $H_{r}$ or not.

Case 2A: The first edge in $p$ after $e_{2}$ is in $H_{r}$. We subdivide $e_{1}=e_{1}^{1} \circ e_{1}^{2}$ such that $\tilde{f}^{\prime}\left(e_{1}^{1}\right)=\tilde{f}\left(e_{2}\right)$ and $\tilde{f}^{\prime}\left(e_{1}^{2}\right)=$ the remaining part of $\tilde{f}\left(e_{1}\right)$. By Property RTT1, $\tilde{f}^{\prime}\left(e_{1}^{1}\right)$ and $\tilde{f}^{\prime}\left(e_{1}^{2}\right)$ are not contained in $X_{r-1}$ and hence the unique exponentially growing stratum that arises from $H_{r}$ is $H_{r}^{\prime}=\left(H_{r} \backslash O_{e_{1}}\right) \cup\left\{O_{e_{1}^{1}}, O_{e_{1}^{2}}\right\}$. We equip $\pi X^{\prime}$ with the length function corresponding to the right eigenvector $l_{r}^{\prime}$ of $M_{r}^{\prime}$ for which $\left\|l_{r}^{\prime}\right\|_{1}=\left\|l_{r}\right\|_{1}$; as a consequence the subdivision preserves length functions. After, we perform the fold $\left(e_{1}^{1}, e_{2}\right)$ and equip $\pi X^{\prime \prime}$ with the length function induced by an appropriate right eigenvector $l^{\prime \prime}$ of $M_{r}^{\prime \prime}$ such that this folding to preserve length functions (by Remark 3.25, it suffices to choose $l_{r}^{\prime \prime}$ such that $\left\|l_{r}^{\prime \prime}\right\|_{1}=\left\|l_{r}\right\|_{1}-l_{r}\left(e_{2}\right)$ ). We note that $\left|H_{r}\right|=\left|H_{r}^{\prime \prime}\right|$ while it is not difficult to see that the map $\tilde{f}^{\prime \prime}$ is a stable relative train track representative of $f$.

Case 2B: The first edge in $p$ after $e_{2}$ is contained in $X_{r-1}$. If $s$ is the maximal subpath of $p_{2}$ that follows $e_{2}$ and is contained in $X_{r-1}$, then $p_{2}=e_{2} \circ s \circ q$ where the first edge of $q$ is in $H_{r}$ and $\tilde{f}\left(p_{2}\right)=\tilde{f}\left(e_{2}\right) \circ \tilde{f}(s) \circ \tilde{f}(q)=t^{-1} \circ p_{2}$. Since $\tilde{f}\left(e_{2}\right)$ is an initial segment of $t^{-1}$ and the first edge of $p_{2}$ is in $H_{r}$, we conclude that $\tilde{f}\left(e_{2}\right) \circ \tilde{f}(s)$ is an initial segment of $t^{-1}$ which is properly contained in $\tilde{f}\left(e_{1}\right)$. As before, we subdivide $e_{1}=e_{1}^{1} \circ e_{1}^{2}$ such that $\tilde{f}^{\prime}\left(e_{1}^{1}\right)=\tilde{f}\left(e_{2} \circ s\right)=\tilde{f}\left(e_{2}\right) \circ \tilde{f}(s)$ and $\tilde{f}^{\prime}\left(e_{1}^{2}\right)=$ the remaining part of $\tilde{f}\left(e_{1}\right)$. Property RTT1 implies that $\tilde{f}^{\prime}\left(e_{1}^{1}\right), \tilde{f}^{\prime}\left(e_{1}^{2}\right) \not \subset$ $X_{r-1}$ and hence the exponentially growing stratum that arises from $H_{r}$ is $H_{r}^{\prime}=$ $\left(H_{r} \backslash O_{e_{1}}\right) \cup\left\{O_{e_{1}^{1}}, O_{e_{1}^{2}}\right\}$. We perform one more subdivision $e_{1}^{1}=e_{1}^{11} \circ e_{1}^{12}$ such that $\tilde{f}^{\prime \prime}\left(e_{1}^{11}\right)=\tilde{f}\left(e_{2}\right)$ and $\tilde{f}^{\prime \prime}\left(e_{1}^{12}\right)=\tilde{f}(s)$. The corresponding exponentially growing stratum is $H_{r}^{\prime \prime}=\left(H_{r}^{\prime} \backslash O_{e_{1}^{1}}\right) \cup\left\{O_{e_{1}^{11}}\right\}$ since $\tilde{f}\left(e_{1}^{12}\right)=\tilde{f}(s) \subseteq X_{r-1}$.

We equip $\pi X^{\prime}$ with the length function as in Case $2 \mathrm{~A}$ and leave the same on $\pi X^{\prime \prime}$, since in this case $M_{r}^{\prime \prime}=M_{r}^{\prime}$. We successively fold $e_{1}^{11}$ with $e_{2}$ and $e_{1}^{12}$ with $s$ (here it is assumed that $e_{1}^{12}$ has been appropriately subdivided). The second fold takes place in $X_{r-1}$ and some nonexponentially growing strata; therefore for the new exponentially growing stratum $H_{r}^{\prime \prime \prime}$ that is produced by $H_{r}^{\prime \prime}$ we have $\left|H_{r}\right|=\left|H_{r}^{\prime \prime \prime}\right|$. We equip $\pi X^{\prime \prime \prime}$ with a length function exactly as $\pi X^{\prime \prime}$ in Case $2 \mathrm{~A}$, because the length of path $s$ is (always) zero. The map $\tilde{f}^{\prime \prime \prime}$ which arises after all these operations is again stable and relative train track.

We remind that the number of edges of $H_{r}$ is reduced by one in Case 1 and remains the same in Case 2 . Thus, by repeating this procedure finitely many times, 
from now on we can suppose that only Case 2 occurs. Finally, we denote by $\left(\tilde{f}^{\prime}, X^{\prime}\right)$ the resulting stable relative train track map and say that $\left(\tilde{f}^{\prime}, X^{\prime}\right)$ is obtained from $(\tilde{f}, X)$ by folding over the indivisible Nielsen path $p$. We also use the symbol ' for the composition of the operations described above. Thus, under the new notation, it is clear that $\left\|l_{r}^{\prime}\right\|_{1}=\left\|l_{r}\right\|_{1}-l_{r}\left(e_{2}\right)$ and $\tilde{f}^{\prime}\left(x^{\prime}\right)=(\tilde{f}(x))^{\prime}$ for each path $x$ of $\pi X$.

Remark 5.1. If the path $q \subseteq X_{r}$ is r-legal (more generally, if it does not contain the illegal turn $\left.g e_{1}^{-1} \circ g e_{2}\right)$ such that its first and last edges are in $H_{r}$, then the first and last edges of $q^{\prime}$ are in $H_{r}^{\prime}$ as well.

Proposition 5.2. Let $f$ be a symmetric automorphism of $G$ and $(\tilde{f}, X)$ a stable relative train track representative of $f$. Let $p$ be an indivisible Nielsen path which is contained in $X_{r}$ such that $p$ intersects the exponentially growing stratum $H_{r}$. Suppose that $e_{1}^{-1} \circ e_{2}$ is the unique illegal turn of $p$ in $H_{r}$ and that $\left(\tilde{f}^{\prime}, X^{\prime}\right)$ is obtained from $(\tilde{f}, X)$ by folding over $p$. Then:

(1) For each path $q$ of $X$ we have $L_{r}^{\prime}\left(q^{\prime}\right)=L_{r}(q)-2 m d$, where $d=\min \left\{l_{r}\left(e_{1}\right), l_{r}\left(e_{2}\right)\right\}$ and $m$ is the number of the turns $g e_{1}^{-1} \circ g e_{2}$ in $q$.

(2) If $q \subseteq X_{r}$ is r-legal, then $q^{\prime}$ is r-legal.

(3) If $q \subseteq X_{r}$ is an indivisible Nielsen path that intersects $H_{r}$, then $q^{\prime} \subseteq X_{r}^{\prime}$ is an indivisible Nielsen path intersecting $H_{r}^{\prime}$ as well.

Proof. The first statement follows readily since the length function $L_{r}^{\prime}$ is defined in such a way that $L_{r}^{\prime}\left(e^{\prime}\right)=L_{r}(e)$ for each edge $e$ of $X_{r}$.

Now, suppose that $q \subseteq X_{r}$ is an r-legal path. Then, by Lemma 4.8, $\tilde{f}^{n}(q)$ is r-legal for all $n \geq 1$. In particular, the turn $g e_{1}^{-1} \circ g e_{2}$ does not occur in $\tilde{f}^{n}(q)$ for any $g \in G$ and $n \in \mathbb{N}$. Therefore $L_{r}^{\prime}\left[\left(\tilde{f}^{n}(q)\right)^{\prime}\right]=L_{r}\left(\tilde{f}^{n}(q)\right)$. By applying Corollary 4.13 we have $L_{r}^{\prime}\left(\left(\tilde{f}^{\prime}\right)^{n}\left(q^{\prime}\right)\right)=L_{r}^{\prime}\left(\left(\tilde{f}^{n}(q)\right)^{\prime}\right)=L_{r}\left(\tilde{f}^{n}(q)\right)=\lambda_{r}^{n} L_{r}(q)=\lambda_{r}^{n} L_{r}^{\prime}\left(q^{\prime}\right)$ for all $n \geq 1$, which implies that $q^{\prime}$ is r-legal and (2) is proved.

We will now prove (3). Let $q=q_{1} \circ q_{2}$ be an indivisible Nielsen path as in (3), where $q_{1}$ and $q_{2}$ are r-legal. Notice that $q^{\prime}$ is fixed by $\tilde{f}^{\prime}$. By Corollary 4.15 the first and last edges of $q_{1}$ and $q_{2}$ are in $H_{r}$. It follows that $q^{\prime}$ intersects $H_{r}^{\prime}$. The path $q^{\prime}$ is the composition of the paths $\left(q_{1}\right)^{\prime}$ and $\left(q_{2}\right)^{\prime}$ which are r-legal because of (2). Therefore $q^{\prime}$ has at most one illegal turn in $H_{r}^{\prime}$.

Suppose, for contradiction, that $q^{\prime}$ is not an indivisible Nielsen path and let $q^{\prime}=q_{1}^{\prime} \circ \cdots \circ q_{n}^{\prime}$ be the decomposition of $q^{\prime}$ into indivisible Nielsen paths. We note that $q$ contains the turn $g e_{1}^{-1} \circ g e_{2}$ at most one being an indivisible Nielsen path. In the case where $q=g e_{2}^{-1} \circ g e_{1} \circ b$ the path $g e_{1} \circ b$ is r-legal and hence $\left(g e_{1}\right)^{\prime} \circ b^{\prime}$ is properly contained in $q_{n}^{\prime}$. It follows that $q^{\prime}=q_{n}^{\prime}$. Otherwise segments of both $\left(q_{1}\right)^{\prime}$ and $\left(q_{2}\right)^{\prime}$ survive in the composition $q^{\prime}=\left(q_{1}\right)^{\prime} \cdot\left(q_{2}\right)^{\prime}$ and since the first and last edges of $\left(q_{i}\right)^{\prime \prime}$ s for $i=1,2$ are in $H_{r}^{\prime}$ (Remark 5.1), we conclude that the first and last edges of $q^{\prime}$ are also in $H_{r}^{\prime}$. Thus $q_{1}^{\prime}$ and $q_{n}^{\prime}$ are indivisible Nielsen paths which intersect $H_{r}^{\prime}$. This means that $q^{\prime}$ must have at least two illegal turns in $H_{r}^{\prime}$ which gives a contradiction.

Remark 5.3. Under the hypotheses of Proposition 5.2 the illegal turn of $p^{\prime}$ is $\left(e_{1}^{2}\right)^{-1}$ 。 $x$, where $x$ is the first edge of $p_{2}$ after $e_{2}$ contained in $H_{r}$. From this we conclude that for every indivisible Nielsen path $q$ the illegal turn of $p^{\prime}$ does not occur in $q^{\prime}$ if the illegal turn of $p$ does not occur in $q$ (see also [10, Theorem IV.4.2]).

In the next theorem we follow the arguments of Bestvina-Handel as these are presented in [10]. 
Theorem 5.4. Suppose that $\tilde{f}: X \longrightarrow X$ is a stable relative train track representative of $f$ and that $H_{r}$ is an exponentially growing stratum. Then there is at most one $G$-orbit of geometric indivisible Nielsen paths in $X_{r}$ that contain edges of $H_{r}$.

Proof. Suppose that $p$ is an indivisible Nielsen path intersecting the stratum $H_{r}$. We consider the sequence $\tilde{f}^{(k)}: X^{(k)} \longrightarrow X^{(k)}$ where $\tilde{f}^{(0)}=\tilde{f}, X^{(0)}=X$ and each $\left(\tilde{f}^{(k+1)}, X^{(k+1)}\right)$ is obtained from $\left(\tilde{f}^{(k)}, X^{(k)}\right)$ by folding at the illegal turn of $p^{(k)}$. We have already seen that $\left|H_{r}\right|=\left|H_{r}^{(k)}\right|$ for every $k$ while by Proposition 5.2 each $p^{(k)}$ is in fact an indivisible Nielsen path that intersects $H_{r}^{(k)}$. Let $\left(e_{1}^{(k)}\right)^{-1} \circ e_{2}^{(k)}$ be the unique illegal turn of $p^{(k)}$ and $d_{k}=\min \left\{l_{r}^{k}\left(e_{1}^{(k)}\right), l_{r}^{k}\left(e_{2}^{(k)}\right)\right\}$, where we denote by $l_{r}^{k}$ the corresponding Perron-Frobenius eigenvector of the matrix $M_{r}^{(k)}$ for all $k$.

We will first show that $L_{r}(p)=2\left\|l_{r}\right\|_{1}$. Proposition 2.3(iii) implies that for the matrices $M_{r}^{(k)}$ there are only a finite number of possibilities. Hence the normalized Perron-Frobenius eigenvectors $\frac{l_{r}^{k}}{\left\|l_{r}^{k}\right\|_{1}}, k \in \mathbb{N}$ are finite as are also their entries; in particular the set $\frac{d_{k}}{\left\|l_{r}^{k}\right\|_{1}}, k \in \mathbb{N}$ is finite. Thus the set $\frac{L_{r}^{k}\left(p^{(k)}\right)}{\left\|l_{r}^{k}\right\|_{1}}, k \in \mathbb{N}$ is a subset of the set consisting of the linear combinations of the form $\sum_{i=1}^{\left|H_{r}\right|} n_{i} r_{i}$ where $n_{i} \in \mathbb{N}$ and $r_{i}$ belong to the finite set of the entries of $\frac{l_{r}^{k}}{\left\|l_{r}^{k}\right\|_{1}}, k \in \mathbb{N}$. Moreover $\frac{L_{r}^{k}\left(p^{(k)}\right)}{\left\|l_{r}^{k}\right\|_{1}}, k \in \mathbb{N}$ is bounded in view of Corollary 4.18. Therefore, it is a finite set.

Now, by Proposition 5.2, $\frac{L_{r}^{k}\left(p^{k}\right)-L_{r}^{k+1}\left(p^{k+1}\right)}{2}=d_{k}=\left\|l_{r}^{k}\right\|_{1}-\left\|l_{r}^{k+1}\right\|_{1}$. Thus, if we suppose that $\frac{L_{r}(p)}{\left\|l_{r}\right\|_{1}} \neq 2$ then $\left|\frac{L_{r}^{1}\left(p^{(1)}\right)}{\left\|l_{r}^{1}\right\|_{1}}-2\right|=\left|\frac{L_{r}(p)-2 d}{\left\|l_{r}\right\|_{1}-d}-2\right|=\left|\frac{L_{r}(p)-2\left\|l_{r}\right\|_{1}}{\left\|l_{r}\right\|_{1}-d}\right|>$ $\left|\frac{L_{r}(p)-2\left\|l_{r}\right\|_{1}}{\left\|l_{r}\right\|_{1}}\right|=\left|\frac{L_{r}(p)}{\left\|l_{r}\right\|_{1}}-2\right| \neq 0$; iterating this we reach to the contradiction that the set $\frac{L_{r}^{k}\left(p^{(k)}\right)}{\left\|l_{r}^{k}\right\|_{1}}$ is infinite. This shows that $\frac{L_{r}(p)}{\left\|l_{r}\right\|_{1}}=2$.

If now, we denote by $p_{1}$ and $p_{2}$ the r-legal parts of $p$ according to Proposition 4.14 , then $L_{r}\left(p_{1}\right)=L_{r}\left(p_{2}\right)=\left\|l_{r}\right\|_{1}$. Of course the preceding arguments work as well for each indivisible Nielsen path $q$ which intersects $H_{r}$. Thus, by working inductively we show that $L_{r}^{k}\left(\left(q^{(k)}\right)_{1}\right)=L_{r}^{k}\left(\left(q^{(k)}\right)_{2}\right)=\left\|l_{r}^{k}\right\|_{1}$ for all $k \in \mathbb{N}$, where $\left(q^{(k)}\right)_{1}$ and $\left(q^{(k)}\right)_{2}$ are the two r-legal parts of $q^{(k)}$.

Since $L_{r}^{k}\left(q^{(k)}\right)-L_{r}^{k+1}\left(q^{(k+1)}\right)=2\left\|l_{r}^{k}\right\|_{1}-2\left\|l_{r}^{k+1}\right\|_{1}=2 d_{k}$, it follows from Proposition 5.2 that the turn $g_{k}\left(e_{1}^{(k)}\right)^{-1} \circ g_{k} e_{2}^{(k)}$ appears in $q^{(k)}$ for some $g_{k} \in G$. Moreover this is the unique illegal turn of $q^{(k)}$. As a consequence we have $\left(p^{(k)}\right)_{1} \cdot\left(g_{k}^{-1} q^{(k)}\right)_{2}=$ $\left(p_{1}\right)^{(k)} \cdot g_{k}^{-1}\left(q_{2}\right)^{(k)}$.

Now, without loss of generality we can suppose that the products $z=p_{1} \cdot g_{0}^{-1} q_{2}$ and $g_{0}^{-1} q_{1} \cdot p_{2}$ are not cancellation free (otherwise we replace $q$ with $q^{-1}$ ). Thus $L_{r}(z)<2\left\|l_{r}\right\|_{1}$. We also note that $z$ has at most one illegal turn since $p_{1}$ and $p_{2}$ are r-legal paths.

Suppose now that $z$ is not a vertex. Then, by Remark 5.1, for each $k$, the path $z^{(k)}$ contains an edge of $H_{r}^{(k)}$ and hence $L_{r}^{k}\left(z^{(k)}\right)>0$ for all $k \in \mathbb{N}$. But $z^{(k)}=\left(p_{1}\right)^{(k)} \cdot g_{0}^{-1}\left(q_{2}\right)^{(k)}=\left(p^{(k)}\right)_{1} \cdot g_{0}^{-1}\left(q^{(k)}\right)_{2}$ and so $L_{r}^{k}\left(z^{(k)}\right) \leq L_{r}^{k}\left(\left(p^{(k)}\right)_{1}\right)+$ $L_{r}^{k}\left(\left(q^{(k)}\right)_{2}\right)=2\left\|l_{r}^{k}\right\|_{1}$.

We claim that

$$
\lim _{k \rightarrow \infty} L_{r}^{k}\left(z^{(k)}\right)=0 .
$$

It suffices to show that $\lim _{k \rightarrow \infty}\left\|l_{r}^{k}\right\|_{1}=0$. The positivity of $d_{k}$ 's implies that the sequence of the positive real numbers $L_{r}^{k}\left(\left(p^{(k)}\right)_{1}\right), k \in \mathbb{N}$, is strictly decreasing converging to its infimum. Therefore the series $\sum_{i=0}^{\infty} d_{i}=L_{r}\left(p_{1}\right)-\lim _{k \rightarrow \infty} L_{r}^{k}\left(\left(p^{(k)}\right)_{1}\right)$ 
converges and so $\lim _{k \rightarrow \infty} d_{k}=0$. As we have seen before the set $\frac{d_{k}}{\left\|l_{r}^{k}\right\|_{1}}$ is finite. It follows that $\lim _{k \rightarrow \infty}\left\|l_{r}^{k}\right\|_{1}=\lim _{k \rightarrow \infty} d_{k} \frac{\left\|l_{r}^{k}\right\|_{1}}{d_{k}}=0$ and this proves the claim.

If there exist $m \geq 0$ and $g \in G$ such that the illegal turn $g\left(\left(e_{1}^{(m)}\right)^{-1} \circ e_{2}^{(m)}\right)$ does not occur in $z^{(m)}$, then we have $L_{r}^{k}\left(z^{(k)}\right)=L_{r}^{m}\left(z^{(m)}\right)$ for all $k \geq m$ in view of Remark 5.3; therefore

$$
\lim _{k \rightarrow \infty} L_{r}^{k}\left(z^{(k)}\right)=L_{r}^{m}\left(z^{(m)}\right)>0
$$

which contradicts the fact that the above sequence converges to zero. This means that for each $k \geq 0$ there is $h_{k} \in G$ such that the illegal turn $h_{k}\left(\left(e_{1}^{(k)}\right)^{-1} \circ e_{2}^{(k)}\right)^{ \pm 1}$ occurs in $z^{(k)}$ exactly one time and hence by Proposition 5.2 we have $L_{r}^{k+1}\left(z^{(k+1)}\right)=$ $L_{r}^{k}\left(z^{(k)}\right)-2 d_{k}$. But now,

$$
\begin{aligned}
L_{r}(z) & =\lim _{k \rightarrow \infty}\left(\sum_{i=0}^{k} 2 d_{i}+L_{r}^{k+1}\left(z^{(k+1)}\right)\right) \\
& =\sum_{k=0}^{\infty} 2 d_{k}=2\left(\left\|l_{r}\right\|_{1}-\lim _{k \rightarrow \infty}\left\|l_{r}^{k}\right\|_{1}\right)=2\left\|l_{r}\right\|_{1}>L_{r}(z)
\end{aligned}
$$

which shows that $z$ must be a vertex. The same arguments can be used to show that $g_{0}^{-1} q_{1}=\left(p_{2}\right)^{-1}$ which yields $g^{-1} q=p^{-1}$ and this completes the proof of the theorem.

Remark 5.5. From the above proof we see that if two indivisible Nielsen paths share the same illegal turn, then they are equal.

\section{The general form of the Scott conjecture}

In this section we give the proofs of what we call the general form of the Scott conjecture and its consequences. We begin with Subsection 6.1 in which we define the notions of the complexity and reduced complexity and state some of their properties.

\subsection{The complexity of a graph of groups.}

Definition 6.1. Let $(\mathcal{G}, Y)$ be a connected graph of groups. We define the complexity $C(Y)$ of $Y$ to be the sum $r(Y)+V_{d}(Y)$, where $V_{d}(Y)$ is the number of the nondegenerate vertices of $Y$. For a graph of groups $(\mathcal{G}, Y)$ with components $Y_{i}, i=1, \ldots, n$, the reduced complexity is

$$
\tilde{C}(Y)=1+\sum_{i=1}^{n} \max \left\{r\left(Y_{i}\right)+V_{d}\left(Y_{i}\right)-1,0\right\}
$$

if $\sum_{i=1}^{n}\left(r\left(Y_{i}\right)+V_{d}\left(Y_{i}\right)\right)>0$ and zero otherwise.

Lemma 6.2 (Monotonicity of the reduced complexity). Let $\Gamma$ be a finite graph of groups and $Y$ a subgraph of $\Gamma$. Then $\tilde{C}(Y) \leq \tilde{C}(\Gamma)$.

Proof. Obviously the conclusion holds if $\tilde{C}(\Gamma)=0$. Proceeding by induction on the number of the connected components of $\Gamma-Y$, it suffices to prove the lemma in the case in which $X=\Gamma-Y$ is connected. Let $Y_{1}, \ldots, Y_{n}$ be the components of $Y, \Lambda$ the subset of $\{1, \ldots, n\}$ such that $C\left(Y_{i}\right)=0$ for $i \in \Lambda$, and $K$ the subset of indexes 
for which the corresponding component intersects $X$. If $\hat{X}$ is the component of $X$ in $\Gamma, n_{i}=\left|V\left(Y_{i}\right) \cap V(X)\right|$, and $d_{i}=\left|V_{d}\left(Y_{i}\right) \cap V_{d}(X)\right|$, then

$$
\begin{aligned}
C(\hat{X})= & r(\hat{X})+V_{d}(\hat{X}) \\
= & E(\hat{X})-V(\hat{X})+1+V_{d}(\hat{X}) \\
= & \sum_{i \in K} E\left(Y_{i}\right)+E(X)-\sum_{i \in K} V\left(Y_{i}\right)-V(X)+\sum_{i \in K} n_{i}+1 \\
& +\sum_{i \in K} V_{d}\left(Y_{i}\right)+V_{d}(X)-\sum_{i \in K} d_{i} \\
= & \sum_{i \in K} E\left(Y_{i}\right)-\sum_{i \in K} V\left(Y_{i}\right)+\sum_{i \in K} V_{d}\left(Y_{i}\right)+E(X)-V(X)+1 \\
& +\sum_{i \in K} n_{i}+V_{d}(X)-\sum_{i \in K} d_{i} \\
= & \sum_{i \in K} C\left(Y_{i}\right)-|K|+C(X)+\sum_{i \in K} n_{i}-\sum_{i \in K} d_{i} \\
= & \sum_{i \in K-\Lambda}\left(C\left(Y_{i}\right)-1\right)+C(X)+\sum_{i \in K-\Lambda}\left(n_{i}-d_{i}\right)+\sum_{i \in K \cap \Lambda}\left(n_{i}-1\right) .
\end{aligned}
$$

We observe that each term in the last equality is a nonnegative integer. In the case where $C(\hat{X})=0$ we have $C\left(Y_{i}\right)-1=C(X)=0$ for each $i \in K-\Lambda$ and consequently $\tilde{C}(\Gamma)=\tilde{C}(Y)$. If now $C(\widehat{X})>0$, then

$$
\begin{aligned}
& \tilde{C}(\Gamma)=+\sum_{i \notin K \cup \Lambda}\left(C\left(Y_{i}\right)-1\right)+(C(\widehat{X})-1) \\
&= 1+\sum_{i \notin K \cup \Lambda}\left(C\left(Y_{i}\right)-1\right)+\sum_{i \in K-\Lambda}\left(C\left(Y_{i}\right)-1\right)+C(X)-1 \\
&+\sum_{i \in K-\Lambda}\left(n_{i}-d_{i}\right)+\sum_{i \in K \cap \Lambda}\left(n_{i}-1\right) \\
&= 1+\sum_{i \notin \Lambda}\left(C\left(Y_{i}\right)-1\right)+C(X)-1+\sum_{i \in K-\Lambda}\left(n_{i}-d_{i}\right)+\sum_{i \in K \cap \Lambda}\left(n_{i}-1\right) \\
&= \tilde{C}(Y)+C(X)-1+\sum_{i \in K-\Lambda}\left(n_{i}-d_{i}\right)+\sum_{i \in K \cap \Lambda}\left(n_{i}-1\right) \\
& \geq \tilde{C}(Y) .
\end{aligned}
$$

The last inequality is clear whenever $n_{i}-d_{i}>0$ for some $i \in K-\Lambda$ or $n_{j}-1>0$ for some $j \in K \cap \Lambda$ or $C(X)>0$. We claim that this is the case. Indeed, we suppose that $C(X)=0, n_{i}-d_{i}=0$ for each $i \in K-\Lambda$ and $n_{j}-1=0$ for each $j \in K \cap \Lambda$. Then, particularly, $d_{i}=0$ for each $i \in K$. On the other hand $n_{i}>0$ for each $i \in K-\Lambda$; therefore $K-\Lambda=\emptyset$. It follows that $C(\hat{X})=0$ which contradicts our assumption that $C(\hat{X})>0$.

Remark 6.3. In the case in which $\Gamma-Y$ consists of only one edge $e$ and $\tilde{C}(Y)>0$, it is elementary to see that $\tilde{C}(\Gamma) \leq \tilde{C}(Y)+1$ (see Figure 1). When $\tilde{C}(Y)=0$ we have $\tilde{C}(\Gamma) \leq 2$. Moreover, if $\tilde{C}(\Gamma)=2$, then either the edge $e$ is a loop with a nondegenerate vertex or an open edge with nondegenerate endpoints. It follows that every finite graph with $m$ edges has complexity at most $m+1$ and exactly $m+1$ when each of its edges has complexity two. 


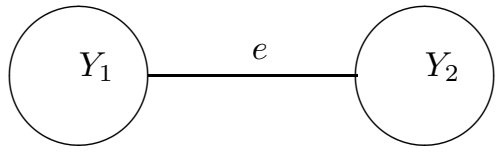

Case 1. $C\left(Y_{1}\right)>0$ and $C\left(Y_{2}\right)>0$
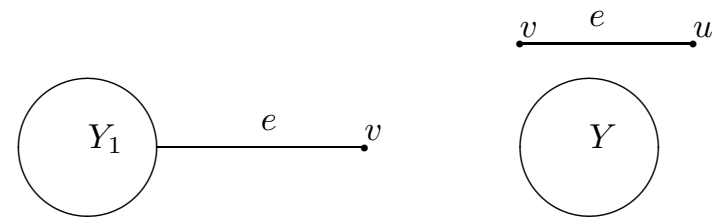

Case 3. $C\left(Y_{1}\right)>0$ and $v$ nondegenerate vertex
Case 4. $u$ and $v$ nondegenerate

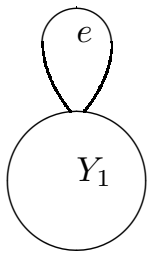

Case 2. $C\left(Y_{1}\right)>0$

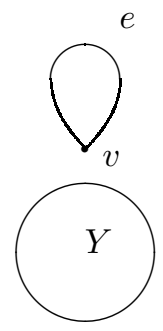

Case 5. $v$ nondegenerate

Figure 1. The five cases in which the adjunction of an edge $e$ on $Y$ with $\tilde{C}(Y)>0$ increases the complexity of $Y$ by one.

Definition 6.4. Let $G$ be a group acting on $X$ and $x \in X$. If we denote by $[x]_{G}$ the orbit of $x$ under the action of $G$, then it is clear that for any subgroup $H$ of $G$ the map $\pi_{G / H}: X / H \longrightarrow X / G$ given by $\pi_{G / H}\left([x]_{H}\right)=[x]_{G}$ is a well-defined map of graphs. Moreover, $\pi_{G / H}$ factors $\pi=\pi_{G / 1_{G}}$ as follows: $\pi=\pi_{G / H} \circ \pi_{H / 1_{G}}$.

Lemma 6.5. Suppose that $G$ acts on a tree $X$ such that all edge groups are finite and have the same cardinality. If $H \leq G$ and $C$ is a noncontractible connected subgraph of $X / H$ (this means that the complexity of $C$ is not zero), then the subgraph $\pi_{G / H}(C)$ of $X / G$ is noncontractible as well.

Proof. Suppose, to the contrary, that $\pi_{G / H}(C)$ is contractible. Then the vertices of $\pi_{G / H}(C)$ and consequently of $C$ are degenerate. Thus, from the noncontractibility of $C$, it follows that $r(C)>0$. Let $p=\left[x_{1}\right]_{H} \circ \cdots \circ\left[x_{n}\right]_{H}$ be a reduced closed path in $C$ such that the path $\pi_{G / H}(p)=\left[x_{1}\right]_{G} \ldots\left[x_{n}\right]_{G}$ is not reduced. Then there is $i \in\{1, \ldots, n\}$ such that $\left[x_{i}\right]_{G}=\left[x_{i+1}\right]_{G}^{-1}$ which means that $x_{i+1}=g x_{i}^{-1}$ for some $g \in G$. Since $t\left(\left[x_{i}\right]_{H}\right)=i\left(\left[x_{i+1}\right]_{H}\right)=i\left(\left[g x_{i}^{-1}\right]_{H}\right)=t\left(\left[g x_{i}\right]_{H}\right)$, it follows that there is $h \in H$ such that the element $h g$ stabilizes the vertex $t\left(x_{i}\right)$. But, the vertices of 
$\pi_{G / H}(C)$ are degenerate; therefore $h g x_{i}=x_{i}$ and hence $\left[x_{i}\right]_{H}=\left[g x_{i}\right]_{H}=\left[x_{i+1}\right]_{H}^{-1}$ which gives a contradiction.

In particular the proof of the above lemma contains the following corollary.

Corollary 6.6. Let $G$ be a group acting on a tree $X$ and $H \leq G$. Suppose that $C$ is a subgraph of $X / H$ such that the graph $\pi_{G / H}(C)$ contains only degenerate vertices. Then the reduced paths of $C$ are mapped via $\pi_{G / H}$ onto reduced paths.

Let $G$ be the fundamental group of a finite graph of groups and $H \leq G$. Then the subgroup $H$ inherits a decomposition as the fundamental group of a graph of groups from this one of $G$ with complexity $C(H)$. The next proposition implies that the complexity $C(H)$ of the subgroup $H$ is invariant under operations described in Section 3.

Proposition 6.7. Let $\pi_{1}(\mathcal{G}, Y)$ and $\pi_{1}\left(\mathcal{G}, Y_{1}\right)$ be two graphs of group decompositions of $G$ which have the same edge and nondegenerate vertex groups and let $\tilde{Y}$ and $\tilde{Y}_{1}$ be the universal trees of $Y$ and $Y_{1}$, respectively. If $H \leq G, \pi_{1}(\mathcal{H}, \tilde{Y} / H)$ and $\pi_{1}\left(\mathcal{H}, \tilde{Y}_{1} / H\right)$ are the graphs of group decompositions where $H$ inherits from these of $G$, then the two sets of the nondegenerate vertices of $\tilde{Y} / H$ and $\tilde{Y}_{1} / H$ can be put into one-to-one correspondence in such a way that corresponding groups of vertices are conjugate; moreover $r(\tilde{Y} / H)=r\left(\tilde{Y}_{1} / H\right)$. In particular, if the graphs $\tilde{Y} / H$ and $\tilde{Y}_{1} / H$ are finite, the two decompositions of $H$ have the same complexity.

Proof. We may assume that the vertices of $\tilde{Y} / H$ and $\tilde{Y}_{1} / H$ constitute sets of representatives of $V(\tilde{Y})$ and $V\left(\tilde{Y}_{1}\right)$, respectively, under the action of $H$. Suppose that $v$ is a nondegenerate vertex of $\tilde{Y} / H$ with corresponding group $H_{v}$. Thus $H_{v}$ is the stabilizer of the vertex $v \in \tilde{Y}$ under the action of $H$ and so $H_{v}=G_{v} \cap H$ for the nondegenerate vertex $v$, under the action of $G$. Since $Y$ and $Y_{1}$ have the same set of nondegenerate vertex groups, there is a unique nondegenerate vertex $u^{\prime}$ of $\tilde{Y}_{1}$ with $G_{u^{\prime}}=G_{v}$. It follows that $H_{v}=H_{u^{\prime}}=h_{u} H_{u} h_{u}^{-1}$, where $h_{u} \in H$ with $u^{\prime}=h_{u} u$ and $u$ is a nondegenerate vertex of $\tilde{Y}_{1} / H$. We can therefore set up a one-to-one correspondence between the two sets of the nondegenerate vertices of $\tilde{Y} / H$ and $\tilde{Y}_{1} / H$ such that corresponding vertex groups are conjugate in $H$.

Suppose now that $v$ is a degenerate vertex of $\tilde{Y}$ (under the action of $H$ ). Then, $H_{v}=H_{e}$ for some edge $e$ of $\tilde{Y}$. Since $Y$ and $Y_{1}$ have the same edge groups, there is an edge $e_{1}$ of $\tilde{Y}_{1}$ such that $G_{e}=G_{e_{1}}$ and hence $H_{v}=H_{e}=G_{e} \cap H=$ $G_{e_{1}} \cap H=H_{e_{1}} \leq H_{i\left(e_{1}\right)}$. Thus, in the case where all vertices of $\tilde{Y} / H$ and $\tilde{Y}_{1} / H$ are degenerate, for each vertex $v$ of $\tilde{Y}$ (resp. $\tilde{Y}_{1}$ ) there is a vertex $u$ of $\tilde{Y}_{1}$ (resp. $\tilde{Y}$ ) such that $H_{v} \leq H_{u}$.

In each case the groups $H_{v}, v \in V(\tilde{Y})$ and $H_{u}, u \in V\left(\tilde{Y}_{1}\right)$ generate the same normal subgroup $N$ of $H$ and the quotient group $H / N$ is isomorphic to the fundamental group of each of the graphs $\tilde{Y} / H$ and $\tilde{Y}_{1} / H$ [9. Proposition I.4.4]. Hence $r(\tilde{Y} / H)=r\left(\tilde{Y}_{1} / H\right)$, and this finishes the proof.

6.2. Main results. We begin by stating some lemmas.

Lemma 6.8. Let $f$ be a symmetric automorphism of $G,(\tilde{f}, X)$ a stable relative train track representative of $f$, and $p, q$ two indivisible Nielsen paths such that $p, q \subseteq X_{r}$. Suppose that $p$ intersects the exponentially growing stratum $H_{r}$ and that $p, q$ have an (internal) vertex in common; then $p=q$. 
Proof. Let $p=p_{1} \circ a \circ p_{2}$ and $q=q_{1} \circ a \circ q_{2}$, where the path $a$ (may be a vertex) is the maximal common segment of $p$ and $q$. Note that from Corollary 4.15 the first and last edges of $p$ belong to $H_{r}$. We claim that $p=a$. This clearly implies that $p=q$.

Assume, on the contrary, that $p \neq a$. Then, in the sequel, without loss of generality we can suppose that $p_{1}$ is not a vertex. We distinguish three cases.

Case 1: The paths $a$ and $p_{2}$ are not vertices. Then, in this case $q_{2}$ is not a vertex, since $p$ is an indivisible Nielsen path. We observe that the paths $p_{1} \circ a \circ p_{2}$, $p_{1} \circ a \circ q_{2}, p_{1} \circ q_{1}^{-1}, q_{2}^{-1} \circ p_{2}$ and $q_{1} \circ a \circ p_{2}$ are indivisible Nielsen paths that intersect $H_{r}$. Hence, by Theorem 5.4 all these paths are in the same $G$-orbit. It follows that $\left|p_{2}\right|=\left|q_{2}\right|,\left|a \circ p_{2}\right|=\left|q_{1}\right|$ and $\left|q_{2}\right|=\left|q_{1} \circ a\right|$, which give the contradiction $\left|q_{2}\right|=\left|q_{1} \circ a\right|>\left|q_{1}\right|>\left|p_{2}\right|=\left|q_{2}\right|$.

Case 2: The path $a$ is not a vertex while the paths $p_{2}$ and $q_{2}$ are vertices. As in the previous case we see that the paths $q_{1}, p_{1}$, and $a^{-1}$ lie in the same $G$-orbit. Since $p$ has a unique illegal turn $e_{1}^{-1} \circ e_{2}$ in $H_{r}$ and $g e_{1}^{-1} \circ g e_{2}$ remains an illegal turn in $H_{r}$ for each $g \in G$, we conclude that the first edge of $a$ must be $e_{2}$ and the last edges of $p_{1}$ and $q_{1}$ are $e_{1}^{-1}$ and $g e_{1}^{-1}$, respectively. Moreover $e_{1}^{-1} \circ g e_{1}$ is the unique illegal turn of $p_{1} \circ q_{1}^{-1}$, which is improper in view of Remark 4.6.

Case 3: The path $a$ is a vertex. In this case we work similarly to Case 2 and obtain a contradiction as well.

Let $f$ be a symmetric automorphism of $G$ and $(\tilde{f}, X)$ a stable relative train track representative of $f$. We define $X_{N}$ to be the subtree of $X$ which consists of all indivisible Nielsen paths. By $X^{N}$ we denote the tree $X_{N}$ when we are thinking each indivisible Nielsen path $p \subseteq X_{r}$ that intersects any exponentially growing stratum $H_{r}$ as an edge. The above lemma ensures that the subgroup Fix $(f)$ acts on $X^{N}$ by isometries. It is easy to see that this action is without inversions. Indeed, suppose, for contradiction, that $p$ is an indivisible Nielsen path as above for which there is an $h \in F i x(f)$ such that $h p=p^{-1}$. We write $p=p_{1} \circ p_{2}$, where $p_{1}$ and $p_{2}$ are the r-legal parts of $p$. Then $h p_{1}$ and $h p_{2}$ are also r-legal parts. Since $h p_{1} \circ h p_{2}=p^{-1}=p_{2}^{-1} \circ p_{1}^{-1}$ and $p_{1}^{-1}, p_{2}^{-1}$ are the r-legal parts of $p^{-1}$, we conclude that $h p_{1}=p_{2}^{-1}$ and $h p_{2}=p_{1}^{-1}$. But, the unique illegal turn of $p$ is $e_{1}^{-1} \circ e_{2}$, where $e_{1}^{-1}$ is the last edge of $p_{1}$ and $e_{2}$ is the first edge of $p_{2}$. It follows that $h e_{1}^{-1}=e_{2}^{-1}$, a contradiction.

Lemma 6.9. Let $H_{r}$ be a stratum whose Perron-Frobenius eigenvalue is 1. Suppose that there are indivisible Nielsen paths in $X_{r}$ that intersect $H_{r}$. Then $H_{r}$ consists of one orbit of edges, let $[e]_{G}$, and if ge is contained in an indivisible Nielsen path in $X_{r}$, then $f(g)^{-1} g \in G_{e}$.

Proof. Let $p=x_{1} \circ \cdots \circ x_{n}$ be an indivisible Nielsen path in $X_{r}$ containing the edges $x_{i_{1}}, \ldots, x_{i_{k}}, i_{j} \in\{1, \ldots, n\}$, of $H_{r}$. By Proposition 2.3, the matrix $M_{r}$ of $H_{r}$ is a permutation matrix. Hence each path $\tilde{f}\left(x_{i_{j}}\right)$ contains exactly one edge of $H_{r}$. It follows that in $p$ there is a segment of $\tilde{f}\left(x_{i_{j}}\right)$ for each $j$ which must contain the edge $x_{i_{j}}$. Thus either $\tilde{f}\left(x_{i_{j}}\right)=x_{i_{j}} \circ q_{j}$ or $\tilde{f}\left(x_{i_{j}}\right)=q_{j} \circ x_{i_{j}}$ for some $q_{j}$ in $X_{r-1}$, since $F \operatorname{Pn} V(\tilde{f})=0$. This means that $H_{r}$ consists of the orbit of an edge. Thus, $H_{r}=[e]_{G}$, where $e=x_{i_{1}}$ and $\tilde{f}(e)=e \circ q_{1}$ or $\tilde{f}(e)=q_{1} \circ e$, for some path $q_{1}$ in $X_{r-1}$. Replacing $e$ by $e^{-1}$, if necessary, we can suppose that $\tilde{f}(e)=e \circ q_{1}$. Now, if $p^{\prime}$ is an indivisible Nielsen path containing $g e$, then, as before, we see that $\tilde{f}(g e)=g e \circ q_{1}^{\prime}$. 
On the other hand, $\tilde{f}(g e)=f(g) e \circ f(g) q_{1}$. It follows that $f(g) e=g e$ and this finishes the proof.

Remark 6.10. If $G$ acts on a tree $X$ such that there are hyperbolic elements (i.e. elements which do not stabilize any vertex of $X$ ), then it is well known that there exists a unique minimal invariant subtree $X^{\prime}$, which consists of the union of the translation axis of all hyperbolic elements of $G$. If $G$ is finitely generated and each element of $G$ stabilizes a vertex of $X$, then $G$ stabilizes a vertex of $X$ and this vertex is the minimal invariant subtree.

Lemma 6.11. Let $G$ be a group acting on a tree $X, f$ a symmetric automorphism of $G$ and $\tilde{f}: X \longrightarrow X$ a representative of $f$. Then $C\left(X_{N} / F i x(f)\right)=C(X / F i x(f))$; that is, the subtree $X_{N}$ is a "core" for Fix $(f)$.

Proof. Let $X^{\prime}$ be the unique minimal invariant Fix $(f)$-subtree of $X$ (if there are no invariant subtrees it follows that $X_{N}=X$ and we set $\left.X^{\prime}=X\right)$. Then $X^{\prime} \subseteq X_{N} \subseteq$ $X$ and hence $r\left(X^{\prime} / F i x(f)\right) \leq r\left(X_{N} / F i x(f)\right) \leq r(X / F i x(f))$. By 9 ] Proposition I.4.4], and the fact that each elliptic element of Fix $(f)$ over $X$ is elliptic and over $X^{\prime}$, it follows that $\operatorname{rank}(X / F i x(f))=\operatorname{rank}\left(X^{\prime} / F i x(f)\right)$ and hence $\operatorname{rank}(X / F i x(f))=$ $\operatorname{rank}\left(X_{N} / F i x(f)\right)$.

Now, since $\tilde{f}(v) \neq v$ for each vertex of $X-X_{N}$, the group Fix $(f) \cap G_{v}$ stabilizes the geodesic $[v, \tilde{f}(v)]$. Thus each vertex of $X-X_{N}$ is degenerate under the action of Fix $(f)$ and this concludes the proof.

We are now ready to prove the first of the main results.

Theorem 6.12. Let $G$ be the fundamental group of a finite graph of groups $Y$ such that all edge groups are finite with the same cardinality, let $|H|$. If $f$ is a symmetric automorphism of $G$ (that is, $f$ maps nondegenerate vertex groups onto conjugates of themselves), then the subgroup Fix $(f)$ inherits from $G$ a splitting of complexity at most $C(Y)|H|$.

Proof. Let $(\tilde{f}, X)$ be a stable relative train track representative of $f$. We first consider the case in which $\tilde{f}$ has no fixed points. Then we can reorient the edges of $X$ such that each vertex of $X$ is the initial vertex of at most one positive edge and by a standard argument we conclude that the quotient graph $X / F i x(f)$ has rank at most one (for details we refer the reader to [10, Lemma I.5.3]). On the other hand, since $\tilde{f}(v) \neq v$ for each vertex of $X$ the group, Fix $(f) \cap G_{v}$ stabilizes the geodesic $[v, \tilde{f}(v)]$ and hence each vertex of $X / F i x(f)$ is degenerate. It follows that $C\left(X^{N} / F i x(f)\right) \leq 1$

So we can suppose that $\tilde{f}$ has fixed points. In this case we denote by $X_{i}^{N}$ the subtree of $X$ consisting of all indivisible Nielsen paths contained in the stratum $X_{i}$ for $i=1, \ldots, n$ (see the comments preceding Lemma 6.9). From Theorem 5.4, if $p$ and $q$ are two indivisible Nielsen paths in the same exponentialy growing stratum, then there is $g \in G$ such that $p=g q$; therefore $f(g) q=g q$ and $f(g)^{-1} g \in G_{q}$. Also, we observe that if $f(g)^{-1} g=f(x)^{-1} x$ for $x \in G$, then the paths $g q$ and $x q$ are in the same Fix $(f)$-orbit. Since $G_{q}$ has at most $|H|$ elements, it follows that in each stratum $H_{i}^{N}$ of $X^{N}$ there are at most $|H| F i x(f)$-orbits of edges. As a consequence the graph $X_{i}^{N} / F i x(f)$ is obtained from $X_{i-1}^{N} / F i x(f)$ by adjoining at most $|H|$ edges, when $H_{i}$ is an exponentially growing stratum. Using Lemma 6.9 
and the above argument, we see that the conclusion also remains true in the case where $H_{r}$ is a stratum with Perron-Frobenius eigenvalue $l_{r}=1$.

We will prove by induction on $i$ that $\tilde{C}\left(X_{i}^{N} / F i x(f)\right) \leq \tilde{C}\left(X_{i} / G\right)|H|$. If $i=1$, then $X_{1}^{N} / F i x(f)$ consists of at most $|H|$ edges and so has reduced complexity at most $|H|+1$. On the other hand, $X_{1} / G$ contains the $G$-orbit of the corresponding indivisible Nielsen path $p$ whose endpoints, in view of Remark 6.3, are nondegenerate vertices if $X_{1}^{N} / F i x(f)$ has reduced complexity $|H|+1$. If $\tilde{C}\left(X_{1}^{N} / F i x(f)\right)=0$, then there is nothing to prove. Now if some component of $X_{1}^{N} /$ Fix $(f)$ is noncontractible, then Lemma 6.5 implies that the corresponding component of $X_{1} / G$ is noncontractible as well. Moreover, Corollary 6.6 for $H=1$ and $C=p$ implies that the component $P$ of $X_{1} / G$ containing $[p]_{G}$ has complexity at least two if $X_{1}^{N} / F i x(f)$ has reduced complexity $|H|+1$. Thus $\tilde{C}\left(X_{1} / G\right)|H| \geq C(P)|H| \geq(1+\epsilon)|H| \geq \tilde{C}\left(X_{1}^{N} / F i x(f)\right)$, where $\epsilon \in\{0,1\}$ and $\epsilon=1$ when $X_{1}^{N} /$ Fix $(f)$ has reduced complexity $|H|+1$.

We next consider the case where $i>1$. If $\tilde{C}\left(X_{i}^{N} / F i x(f)\right)=\tilde{C}\left(X_{i-1}^{N} / F i x(f)\right)$, then $\tilde{C}\left(X_{i}^{N} / F i x(f)\right)=\tilde{C}\left(X_{i-1} / G\right) \leq \tilde{C}\left(X_{i} / G\right)|H|$. Now suppose that

$$
\tilde{C}\left(X_{i}^{N} / F i x(f)\right)>\tilde{C}\left(X_{i-1}^{N} / F i x(f)\right) .
$$

Let $\left[e_{1}\right]_{F i x(f)}, \ldots,\left[e_{k}\right]_{F i x(f)}, k \leq|H|$ be the edges (in fact the Fix $(f)$-orbits of all indivisible Nielsen paths intersecting $\left.H_{r}\right)$ of $H_{i}^{N}$ whose adjunction on $X_{i-1}^{N} / F i x(f)$ increases the reduced complexity. We have already seen that $\left[e_{1}\right]_{F i x(f)}, \ldots,\left[e_{k}\right]_{F i x(f)}$ belong to the same $G$-orbit, let $[p]_{G}$. This means that $\pi_{G / F i x(f)}\left(\left[e_{i}\right]_{F i x(f)}\right)=[p]_{G}$, for each $i=1, \ldots, k$. We distinguish two cases.

Case 1: There exists some endpoint of $[p]_{G}$, let $t[p]_{G}$, which is degenerate and belongs to a contractible component of $X_{i-1} / G$ when $t[p]_{G} \in X_{i-1} / G$. Then the following hold:

(1) For each $i=1, \ldots, k$ the vertices $t\left[e_{i}\right]_{F i x(f)}$ are degenerate and, by Lemma 6.5 , those that are contained in $X_{i-1}^{N} / F i x(f)$ belong to contractible components of $X_{i-1}^{N} / F i x(f)$.

(2) In $X_{i}^{N} / F i x(f)$ there are no paths of the form $\left[e_{i}\right]_{F i x(f)} \circ$ $\left[e_{j}\right]_{F i x(f)}^{-1}$. Indeed, suppose that there exists a path of the form $\left[e_{i}\right]_{F i x(f)} \circ\left[e_{j}\right]_{F i x(f)}^{-1}$. Then $t\left[e_{i}\right]_{F i x(f)}=i\left[e_{j}\right]_{F i x(f)}^{-1}=$ $t\left[g e_{i}\right]_{F i x(f)}$ for some $g$ in $G$ and so $t\left(e_{i}\right)=h g t\left(e_{i}\right)$ for some $h$ in Fix $(f)$. Since, in this case, the vertex $t\left(e_{i}\right)$ is degenerate (under the action of $G$ ), it follows that $e_{i}=h g e_{i}$ and consequently $\left[e_{i}\right]_{F i x(f)}=\left[e_{j}\right]_{F i x(f)}$, a contradiction to our assumptions.

(3) If the vertices $t\left[e_{i}\right]_{F i x(f)}$ and $t\left[e_{j}\right]_{F i x(f)}$ for $i \neq j$ are in the same component $A$ of $X_{i-1}^{N} /$ Fix $(f)$, then they must be equal. In order to prove this consider the path $z$ in $A$ from $t\left[e_{i}\right]_{F i x(f)}$ to $t\left[e_{j}\right]_{F i x(f)}$ and assume that $z$ is not trivial. The component $A^{\prime}$ of $X_{i-1} / G$ containing $\pi_{G / F i x(f)}(A)$ is contractible since it also contains the vertex $t[p]_{G}$. By Corollary $6.6, \pi_{G / F i x(f)}(z)$ is a reduced closed path, which contradicts the contractibility of $A^{\prime}$. Hence, by item (2), in each component $A$ of $X_{i-1}^{N} / F i x(f)$ there is at most one incoming edge of $\left[e_{1}\right]_{F i x(f)}, \ldots,\left[e_{k}\right]_{F i x(f)}$. 
Let $\hat{\Gamma}$ be a component of $X_{i}^{N} / F i x(f)$ containing edges of

$$
\Gamma=\left\{\left[e_{1}\right]_{F i x(f)}, \ldots,\left[e_{k}\right]_{F i x(f)}\right\},
$$

and let $A_{1}, \ldots, A_{\nu}$ be the components of

$$
X_{i-1}^{N} / F i x(f) \cup\left\{i\left[e_{i}\right]_{F i x(f)}, t\left[e_{i}\right]_{F i x(f)}, i=1, \ldots, k\right\}
$$

in $\hat{\Gamma}$.

If all components $A_{i}$ for $i=1, \ldots, \nu$ are contractible, then, as the reader can easily verify, $C(\hat{\Gamma})=r(\hat{\Gamma}) \leq 1$, since for each component $A_{i}$ there exists at most one incoming edge $\left[e_{i}\right]_{F i x(f)}$ (item (3)).

Suppose now that there exists a noncontractible component $A_{i_{0}}, i_{0} \in\{1, \ldots, \nu\}$. In this case, we will show that $C(\hat{\Gamma})=C\left(A_{i_{0}}\right)$. First, we note that each component $A_{i}$ for $i \neq i_{0}$ is contractible. Indeed, if $q$ is a path in $\hat{\Gamma}$ which connects the components $A_{i_{0}}$ and $A_{i}$, then we write $q=q_{0} \circ\left[e_{i_{0}}\right]_{F i x(f)}^{\epsilon_{0}} \circ q_{1} \circ\left[e_{i_{1}}\right]_{F i x(f)}^{\epsilon_{1}} \circ$ $\cdots \circ\left[e_{i_{m}}\right]_{F i x(f)}^{\epsilon_{m}} \circ q_{m+1}$, where $\epsilon_{i} \in\{ \pm 1\},\left[e_{i_{j}}\right]_{F i x(f)} \in \Gamma, q_{i} \cap E(\Gamma)=\emptyset, q_{0} \subseteq A_{i_{0}}$, and $q_{m+1} \subseteq A_{i}$. Since $A_{i_{0}}$ is a noncontractible component, it follows that $\epsilon_{0}=1$ (item (1)). Thus, by item (3), $\epsilon_{1}=\cdots=\epsilon_{m}=1$ and therefore $t\left[e_{i_{m}}\right]_{F i x(f)}$ belongs to $A_{i}$. This shows that $A_{i}$ is contractible for $i \neq i_{0}$. Taking $A_{i}$ to be any nondegenerate vertex of $\hat{\Gamma}$, we see that the preceding argument shows that all nondegenerate vertices of $\hat{\Gamma}$ are contained in $A_{i_{0}}$. Thus, to prove our assertion (i.e. $\left.C(\hat{\Gamma})=C\left(A_{i_{0}}\right)\right)$ it suffices to show that if $q$ is a reduced closed path in $\hat{\Gamma}$ starting and ending at some vertex of $A_{i_{0}}$, then it is contained in $A_{i_{0}}$. To show this, suppose on the contrary that $q$ is not contained in $A_{i_{0}}$. Then, as before, we write $q=q_{0} \circ\left[e_{i_{0}}\right]_{F i x(f)}^{\epsilon_{0}} \circ q_{1} \circ\left[e_{i_{1}}\right]_{F i x(f)}^{\epsilon_{1}} \circ \cdots \circ\left[e_{i_{m}}\right]_{F i x(f)}^{\epsilon_{m}} \circ q_{m+1}$, where $\epsilon_{i} \in\{ \pm 1\}$, $\left[e_{i_{j}}\right]_{F i x(f)} \in \Gamma, q_{i} \cap E(\Gamma)=\emptyset$, and $q_{0}, q_{m+1} \subseteq A_{i_{0}}$. The noncontractibility of $A_{i_{0}}$ gives $\epsilon_{0}=1$, from which it follows that $\epsilon_{1}=\cdots=\epsilon_{m}=1$. In particular $A_{i_{0}}$ contains the vertex $t\left[e_{i_{m}}\right]_{F i x(f)}$. Therefore $A_{i_{0}}$ must be contractible, a contradiction.

From the preceding analysis, we deduce that in order to have $\tilde{C}\left(X_{i-1}^{N} / F i x(f)\right)<$ $\tilde{C}\left(X_{i}^{N} / F i x(f)\right)$ the complexity of each component of $X_{i-1}^{N} / F i x(f)$ must be zero. This means that the reduced complexity of $X_{i-1}^{N} / F i x(f)$ is zero. Hence the reduced complexity of $X_{i}^{N} / F i x(f)$ is at most $|H|$ and the conclusion follows as in the inductive step for $i=1$.

Case 2: The endpoints $i[p]_{G}$ and $t[p]_{g}$ of $[p]_{G}$ are in noncontractible components (or in the same noncontractible component) of $X_{i-i} / G$, provided that a component may be an endpoint of $[p]_{G}$. In this case we have $\tilde{C}\left(X_{i-1} / G\right)+1 \leq \tilde{C}\left(X_{i} / G\right)$. This follows directly from the monotonicity of the reduced complexity plus Corollary 6.6 when the vertices of $[p]_{G}$ which are not contained in $X_{i-1} / G$ are degenerate. We distinguish the following two subcases.

Case 2A: $\tilde{C}\left(X_{i}^{N} / F i x(f)\right)=\tilde{C}\left(X_{i-1}^{N} / F i x(f)\right)+|H|+1$. In this case, Remark 6.3 implies that $\tilde{C}\left(X_{i-1}^{N} / F i x(f)\right)=0$ and that the endpoints of $[p]_{G}$ are nondegenerate vertices. Now, it is not difficult to check that $\tilde{C}\left(X_{i} / G\right) \geq 2$ and thus $\tilde{C}\left(X_{i}^{N} / F i x(f)\right)=|H|+1 \leq \tilde{C}\left(X_{i} / G\right)|H|$.

Case 2B: $\tilde{C}\left(X_{i}^{N} / F i x(f)\right) \leq \tilde{C}\left(X_{i-1}^{N} / F i x(f)\right)+|H|$. Then $\tilde{C}\left(X_{i}^{N} / F i x(f)\right) \leq$ $\tilde{C}\left(X_{i-1}^{N} / F i x(f)\right)+|H| \leq \tilde{C}\left(X_{i-1} / G\right)|H|+|H|=\left(\tilde{C}\left(X_{i-1} / G\right)+1\right)|H| \leq \tilde{C}\left(X_{i} / G\right)|H|$ and this completes the proof of the inductive step.

Finally, $C\left(X^{N} / F i x(f)\right) \leq C(X / G)|H|$ since the graphs $X_{n} / G=X / G$ and $X_{n}^{N} / F i x(f)=X^{N} / F i x(f)$ are connected. On the other hand, each vertex $v$ in the 
interior of an indivisible Nielsen path $p$ is not fixed by $\tilde{f}$; it follows that $[v]_{\text {Fix }(f)}$ is degenerate. These together with Proposition 3.29, Proposition 6.7 and Lemma 6.11 imply that $C(\tilde{Y} / F i x(f)) \leq C(Y)|H|$, where $\tilde{Y}$ denotes the universal tree of $Y$, and this finishes the proof.

We now state some special but interesting cases of Theorem 6.12 .

Proposition 6.13. Let $G$ be the fundamental group of the union $Y$ of $n$ circles with a single point in common such that all edge groups have the same cardinality and the vertex group has at most one end (resp. finite). If $f$ is any automorphism (resp. monomorphism) of $G$, then $f$ is symmetric and $C(\tilde{Y} / F i x(f)) \leq n|H|$ or $(n+1)|H|$, depending on whether the vertex is degenerate or not.

Proof. In view of Theorem 6.12 and Remark 3.3, it suffices to show that $f$ is symmetric. We first note that the corresponding universal tree $\tilde{Y}$ has one orbit of vertices. Thus if $G_{v}$ is the vertex group, then since $G_{v}$ is one-ended so is $f\left(G_{v}\right)$ and hence $f\left(G_{v}\right)$ stabilizes a vertex $g v$ of $\tilde{Y}$. That is, $f\left(G_{v}\right) \subseteq g G_{v} g^{-1}$. It is clear that the above inclusion is equality if the vertex $v$ is degenerate and more generally finite. In the case that $v$ is nondegenerate, the same argument, with $f^{-1}$ instead of $f$, shows that $f^{-1}\left(G_{v}\right) \subseteq x G_{v} x^{-1}$ and finally $G_{v} \subseteq f(x) f\left(G_{v}\right) f\left(x^{-1}\right) \subseteq$ $f(x) g G_{v} g^{-1} f\left(x^{-1}\right)$. Since $v$ is nondegenerate we have $f\left(G_{v}\right)=g G_{v} g^{-1}$ and this proves that $f$ is symmetric.

Corollary 6.14 (2], 10]). Let $f$ be a monomorphism of a free group $F_{n}$ of rank $n$. Then, $\operatorname{rank}(F i x(f)) \leq n$.

Proposition 6.15. Let $G=*_{H} G_{i}$ be the free product of $G_{i}$ 's, $i=1, \ldots, n$, with amalgamated subgroup $H$ properly contained in each factor $G_{i}$. Suppose that $H$ is a finite group while each factor $G_{i}$ has at most one end. If $f \in \operatorname{Aut}(G)$, then the subgroup Fix $(f)$ inherits from $G$ a splitting of complexity at most $n|H|$.

Proof. It is well known that $G$ is the fundamental group of a tree of groups $(\mathcal{G}, T)$ with $n+1$ edges and $n+2$ vertices such that each edge group is $H$ and the vertex groups are $H, G_{1}, \ldots, G_{n}$. Since each factor has at most one end, the standard argument as it is described in the proof of the above proposition shows that $f$ is symmetric and Theorem 6.12 is applied.

Remark 6.16. The conclusion of Proposition 6.15 remains valid when each factor is indecomposable over finite groups $K$ with $|K| \leq|H|$. There are many cases in which this happens. For example each group $A *_{\Gamma} B$, where $A, B$, and $\Gamma$ are finite groups with $|\Gamma|>|H|$, has this property, as the reader can easily verify.

Thus in the case of free products it suffices to suppose that each factor not isomorphic to $\mathbb{Z}$ is indecomposable with respect to free products in order to have $f$ symmetric. In this case the corresponding graph of groups is obtained from $(\mathcal{G}, T)$ by attaching one loop for each infinite cyclic factor.

Corollary 6.17 ([8]). Let $G=\boldsymbol{*}_{i}^{n} G_{i}$ be the free product of $G_{i}$ 's, $i=1, \ldots, n$, where each factor $G_{i}$ is indecomposable with respect to free products and let $f$ be an automorphism of $G$. Then the subgroup Fix $(f)$ has complexity at most $n$.

Theorem 6.12 , together with the accessibility of finitely presented groups and Stallings' ends theorem, allows us to prove the following theorem. 
Theorem 6.18. Let $G$ be a finitely presented group with infinite ends and let $(\mathcal{G}, Y)$ be a graph of groups decomposition of $G$ of minimal number of vertices such that all vertex groups have at most one end and all edge groups are finite. If $f \in A u t(G)$, then there is a positive integer $n=n(G)$ such that Fix $(f)$ admits a graph of groups decomposition with vertex and edge groups of the form Fix $(f) \cap G_{x}, g \in G, x \in Y$, of complexity at most $n$.

Proof. We first note that since finitely presented groups are accessible, $G$ is the fundamental group of a finite graph of groups such that all vertex groups have at most one end and all edge groups are finite; thus we can choose a graph of groups with fundamental group $G$ as in the statement of the theorem. We proceed by induction on the number of the nonsame cardinality edge groups. If this number is one, then the above results apply. Suppose now that this number is at least two. Let $Y_{\min }=\left\{e \in E(Y):\left|G_{e}\right|\right.$ is minimal $\}$ and let $Y_{1}, \ldots, Y_{k}$ be the components of $Y \backslash Y_{\min }$. By contracting each component $Y_{i}$ to a point, we take a graph of groups $(\mathcal{G}, Z)$ with fundamental group $G$ such that all edge groups have the same cardinality $\left|G_{e}\right|, e \in Y_{\text {min }}$, and each vertex group $G_{v(i)}$ is the fundamental group of the component $Y_{i}$. In particular each vertex $v(i)$ of $Z$ is nondegenerate. We will show that $f$ is symmetric with respect to the splitting $(\mathcal{G}, Z)$ of $G$.

We claim that if $e$ is an edge of $Y \backslash Y_{\min }$ with $i(e) \neq t(e)$, then the group $\left\langle G_{i(e)} \cup G_{t(e)}\right\rangle$ generated by $G_{i(e)}$ and $G_{t(e)}$ stabilizes a unique vertex acting on the universal tree $\tilde{Z}$ of $(\mathcal{G}, Z)$. Indeed, it is clear that each group of $G_{i(e)}$ and $G_{t(e)}$ stabilizes a unique vertex of $\tilde{Z}$. If $G_{i(e)}$ and $G_{t(e)}$ do not stabilize the same vertex, then their intersection $G_{e}=G_{i(e)} \cap G_{t(e)}$ stabilizes a geodesic, contradicting the minimality of the edge groups of $Z$. Similarly, if $i(e)=t(e)$, the group $\left\langle G_{i(e)}, g_{e}\right\rangle$ generated by $G_{i(e)}$ and $g_{e}$, where $g_{e} i(e)=t(e)$, stabilizes a unique vertex acting on $\tilde{Z}$. To see this, note that in this case the subgroup $G_{e}$ is the intersection $G_{i(e)} \cap g_{e} G_{i(e)} g_{e}^{-1}$. As before we conclude that the groups $G_{i(e)}$ and $g_{e} G_{i(e)} g_{e}^{-1}$ must stabilize the same unique vertex of $\tilde{Z}$. Thus, $g_{e}$ must also stabilize this vertex, which proves our assertion.

Since each group $f\left(G_{v(i)}\right)$ is constructed from the vertex groups $f\left(G_{v}\right), v \in$ $V\left(Y_{i}\right)$, and the corresponding edge groups, by using amalgamated free products and HNN-extensions and each $f\left(G_{v}\right)$ as before stabilizes a unique vertex of $\tilde{Z}$ having at most one end, the above claim implies that $f\left(G_{v(i)}\right)$ stabilizes a unique vertex as well. Now, the symmetricity of $f$ with respect to $(\mathcal{G}, Z)$ follows by the usual argument. By Theorem 6.12 the subgoup Fix $(f)$ inherits a splitting of bounded complexity from $(\mathcal{G}, Z)$, where the nondegenerate vertex groups are of the form $F i x\left(\left.f\right|_{G_{g v(i)}}\right)$ for $g \in G$ and $g v(i) \in F i x(\tilde{f})$. On the other hand, the fact that $f$ is symmetric means that the restriction $\left.f\right|_{G_{g v(i)}}$ of $f$ is an automorphism. This together with the inductive hypothesis completes the proof of Theorem 6.18.

We recall that $d(G)$ denotes the minimum number of generators of the finitely generated group $G$.

Remark 6.19 . Let $H$ be a polycyclic by finite group, $N$ a normal polycyclic subgroup of $H$ of finite index and $K$ a subgroup of $H$. If $N$ has a polycyclic series with $n$ factors, then $K \cap N$ inherits a polycyclic series with $m \leq n$ factors. Thus $d(K) \leq m|K / K \cap N| \leq n|H / N|$. 
Corollary 6.20. Let $G$ be the fundamental group of a finite graph of groups $(\mathcal{G}, Y)$ such that all vertex groups are polycyclic by finite and all edge groups are finite. Then there exists a positive integer $m(G)$ such that $d(F i x(f)) \leq m(G)$ for each automorphism $f$ of $G$.

Proof. The group $G$, being finitely presented, admits a graph of groups decomposition $(\mathcal{G}, Z)$ such that all vertex groups have at most one end and all edge groups are finite. Since each vertex group $G_{v}, v \in Z$, has at most one end, it stabilizes a vertex acting on the universal tree of $Y$. Therefore $G_{v}$ is polycyclic by finite for each $v \in Z$. Now, the proof follows from Theorem 6.18 and Remark 6.19.

\section{REFERENCES}

[1] H. Bass, Group actions on non-archimedean trees, Arboreal group theory (R.C. Alperin, ed.), M.S.R.I. publ. vol. 19, Springer-Verlag, New York, (1991), pp. 69-131. MR 93d:57003

[2] M. Bestvina and M. Handel, Train tracks and automorphisms of free groups, Ann. of Math. (2) 135 (1992), 1-51. MR 92m:20017

[3] M. Bestvina, M. Feighn and M. Handel, The Tits alternative for Out $\left(F_{n}\right)$ I: Dynamics of exponentially -growing automorphisms, Ann. of Math. (2) 151 (2000), 517-623. MR 2002a:20034

[4] M. Bestvina, M. Feighn and M. Handel, The Tits alternative for Out $\left(F_{n}\right)$ II: A Kolchin type theorem, preprint.

[5] M. Bestvina, M. Feighn and M. Handel, The Tits alternative for Out $\left(F_{n}\right)$ III: Solvable subgroups of $\operatorname{Out}\left(F_{n}\right)$ are virtually abelian, preprint.

[6] D. E. Cohen, Combinatorial Group Theory: a topological approach, London Math. Soc. Stud. Texts 14, Cambridge Univ. Press, Cambridge, 1989. MR 91d:20001

[7] D. J. Collins and E. C. Turner, Free product fixed points, J. London Math. Soc. (2) 38 (1988), 67-76. MR 89h:20037

[8] D. J. Collins and E. C. Turner, Efficient representatives for automorphisms of free products, Michigan Math. J. 41 (1994), 443-464. MR 95k:20039

[9] W. Dicks and M. J. Dunwoody, Groups acting on graphs, Cambridge Univ. Press, 1989. MR 91b:20001

[10] W. Dicks and E. Ventura, The group fixed by a family of injective endomorphisms of a free group, Contemp. Math. 195 (1996), 1-81. MR 97h:20030

[11] S. Gersten, Fixed points of automorphisms of free groups, Adv. in Math. 64 (1987), 51-85. MR 88f:20042

[12] R. Z. Goldstein and E. C. Turner, Fixed subgroups of homomorphisms of free groups, Bull. London Math. Soc. 18 (1986), 468-470. MR 87m:20096

[13] S. Krstic, Fixed subgroups of automorphisms of free by finite groups: an extension of Cooper's proof, Arch. Math. (Basel) 48 (1987), 25-30. MR 88d:20044

[14] J.-P. Serre, Trees, Springer-Verlag, New York, 1980. MR 82c:20083

[15] M. Sykiotis, Fixed points of symmetric endomorphisms of groups, Internat. J. Algebra Comput. (5) 12 (2002), 737-745.

Department of Mathematics, University of Athens, Athens 15784, Greece

Current address: Amalthias 18, Larisa 41222, Greece

E-mail address: msikiot@cc.uoa.gr 